Review Article

\title{
Optimization and Efficiency Studies of Heat Engines: A Review
}

\author{
Preety Aneja
}

Assistant Professor, Department of Physics, DAV College, Jalandhar, Punjab, India.

DOI: https://doi.org/10.24321/2454.8650.202006

\section{I $\quad \mathbf{N} \quad \mathbf{F} \quad \mathbf{O}$}

E-mail Id:

anejapreety2009@gmail.com

Orcid Id:

https://orcid.org/0000-0003-3916-5219

How to cite this article:

Aneja P. Optimization and Efficiency Studies of Heat Engines: A Review. J Adv Res Mech Engi Tech 2020; 7(3): 37-58.

Date of Submission: 2020-07-06

Date of Acceptance: 2020-08-18
$\begin{array}{llllllll}\mathbf{A} & \mathbf{B} & \mathbf{S} & \mathbf{T} & \mathbf{R} & \mathbf{A} & \mathbf{C} & \mathbf{T}\end{array}$

This review aims to study the various theoretical and numerical investigations in the optimization of heat engines. The main focus is to discuss the procedures to derive the efficiency of heat engines under different operating regimes (or optimization criteria) for different models of heat engines such as endreversible models, stochastic models, low-dissipation models, quantum models etc. Both maximum power and maximum efficiency operational regimes are desirable but not economical, so to meet the thermo-ecological considerations, some other compromise-based criteria have been proposed such as $\Omega$ criterion (ecological criterion) and efficient power criterion. Thus, heat engines can be optimized to work at an efficiency which may not be the maximum (Carnot) efficiency. The optimization efficiency obtained under each criterion shows a striking universal behaviour in the near-equilibrium regime. We also discussed a multi-parameter combined objective function of heat engines. The optimization efficiency derived from the multi-parameter combined objective function includes a variety of optimization efficiencies, such as the efficiency at the maximum power, efficiency at the maximum efficiency-power state, efficiency at the maximum criterion, and Carnot efficiency. Thus, a comparison of optimization of heat engines under different criteria enables to choose the suitable one for the best performance of heat engine under different conditions.

Keywords: Optimization Criterion, Operating Regime, Ecological, Efficient Power

\section{Introduction}

The second law of thermodynamics constraints the operation of a heat engine. It sets limits on the possible efficiency of the engine and thus determines the direction of energy flow. Sadi Carnot, a French engineer, in 1826, found that the maximum efficiency of a heat engine operating between a hot and a cold reservoir is attained only for a reversible process and named as Carnot efficiency, ${ }^{1} \eta_{c}=1$ $T_{d} / T_{h^{\prime}}$, with $T_{C^{\prime}}$ and $T_{h}$ being the temperatures of the cold and hot reservoirs respectively and hence, depends exclusively on these temperatures. ${ }^{2}$ Thus, the Carnot efficiency is upper bound for the efficiency of any heat engine. However, Carnot efficiency can only be achieved through an infinitely slow process as required by thermodynamic equilibrium. So, for such a process, the power output resulting from finite heat transfer by heat exchangers should be practically zero. Thus, Carnot efficiency does not seem to have practical importance and constitutes a poor guide for the performance of real heat engines. In recent years, 
several studies have been made in connection with the efficiency of realistic heat engines. The field of "Finite-time thermodynamics" has become a popular area of research since it deals with the realistic constraints in heat engines such as finite-time of the engine operation cycle, finite reservoirs, internal friction etc. These constraints, in turn, lead to a less efficient heat engine than Carnot engine but is of practical importance. The important thing is to find the best mode of operation of heat engines, under more realistic conditions than reversible ones. A number of different optimization criteria have been proposed for heat engines working under finite-time conditions. ${ }^{3-13}$ Some of them maximize power, work, effectiveness, of profit, and there are also those that minimize the loss of available work or entropy production. For practical purposes and to achieve a non-vanishing power output, the Carnot cycle should be speeded up and completed in a finite time.

One optimization criterion widely used is MP criterion to determine the Efficiency at Maximum Power (EMP). This problem has attracted much attention. ${ }^{14-37}$ In 1955, Odum and Pikerton, for the first time, studied the efficiency of heat engine which is made to operate at maximum output power. ${ }^{14}$ Later in 1975 , a better approximation of the performance of real heat engines was made by Curzon and Ahlborn,one of the first models that presents the power output as an optimization criterion and thus derived the efficiency of a heat engine known as the Curzon-Ahlborn (CA) efficiency, $\eta_{C A}=1-\sqrt{T_{c} / T_{h}} \cdot{ }^{3}$ Curzon and Ahlborn considered an irreversible model of heat engine unlike the reversible Carnot model by taking irreversible linear finite rate heat transfer between the working fluid and its two heat reservoirs, though it is not the only source of irreversibility in real heat engines. The Curzon and Ahlborn engine does not allow for any internal irreversibility and so is called "Endoreversible heat engine". ${ }^{15-17}$ The impact of some other kinds of irreversibility on the performance efficiency of real heat engines has been studied by different groups. Subsequently, Gutowicz-Krusin, ${ }^{18}$ Orlov, ${ }^{19}$ DeVos, ${ }^{20,21}$ $\mathrm{Yan}^{22,23}$ studied the irreversible Carnot like heat engines for EMP under different heat transfer laws unlike Newton heat transfer law used by Curzon and Ahlborn. Gordon ${ }^{24}$ made an attempt to incorporate internal irreversibility in the finite-time analysis of the power vs efficiency relationship of a thermoelectric generator and later the internal irreversibilities in a Carnot engine were characterized in terms of entropy generations ${ }^{25}$ to study the performance of heat engine at maximum power. The validity of $\eta_{C A}$ as an upper bound for heat engines, as well as its universal character, were the subject of a long standing debate. In the linear regime, it was shown that the efficiency at maximum power is indeed limited by the Curzon-Ahlborn efficiency, which in this regime is exactly half of the Carnot efficiency. ${ }^{26}$ Later, Schmiedl and Seifert, ${ }^{29} \mathrm{Tu}_{\mathrm{ZC}}{ }^{30}$ and Esposito et al. ${ }^{31}$ investigated the problem of EMP using stochastic heat engines, Feynmann's ratchet and quantum dot engines. The corresponding thermodynamic efficiency in all these models agrees with the $\eta_{C A}$ up to quadratic terms in $n_{c}$. One of the most profound findings is the universal behaviour of the EMP, ${ }^{32}$ i.e., at small relative temperature differences the EMP can be universally expressed in terms of the Carnot efficiency up to quadratic order, $\eta_{M P}=\eta_{C} / 2+\eta_{C}^{2} / 8+O\left(\eta_{C}^{3}\right)$ where the linear coefficient $1 / 2$ is universal for the systems operating under the strong coupling condition in thelinear response regime. ${ }^{26}$ Beyond the linear response, the universal value of the quadratic coefficient isequal to $1 / 8$ for the strong coupling systems in the presence of leftright symmetry. ${ }^{32}$ Esposito et al. ${ }^{34}$ found that the EMP for low-dissipation Carnot-like engines was bounded between $\eta_{-} \equiv \eta_{C} / 2$ and $\eta_{+} \equiv \eta_{C} /\left(2-\eta_{C}\right)$. All these results have been confirmed within a minimally nonlinear irreversible thermodynamics framework. ${ }^{37-39}$ Subsequently, Uzdin and Kosloff ${ }^{40}$ studied hot quantum Otto engines and identified the universal behaviour of the efficiency at maximum output power. Sheng and $\mathrm{Tu}^{41}$ applied the generic finite-time thermodynamics to obtain the universality of the EMP for tight-coupling heat engines. Cleuren et al. ${ }^{42}$ demonstrated how symmetries and constraints at the microscopic level, combined with the fluctuation theorem, emerge at the macroscopic level via the expression for the EMP. The universality of EMP has been extensively investigated in literature. However, the actual thermal plants and heat engines may not work in maximum power regime but rather in the regime with slightly small power and considerably large efficiency, Therefore, it is of great importance to study the efficiency of heat engines at arbitrary power output. The studies in this direction were performed in Refs. [43-49] In above models, $\eta_{C A}$ seems to have some sort of universality independent of the model details. But, in CA efficiency, the temperature differences between the reservoirs and the working substance are taken as the parameters to maximize the power, they do not seem easily controllable. Thus, the CA efficiency seems to be still controversial in these aspects. Regarding the verification of the validity of $\eta_{C A}$, Izumida and Okuda (IO $)^{50,51}$ proposed numerical experiments performed by means of a Molecular Dynamics (MD) simulation ${ }^{52-54}$ of a weakly interacting gas, which can be regarded as a nearly ideal gas, in a finite-time Carnot cycle. The authors studied the efficiency at maximum power $\left(\eta_{\text {MP }}\right)$ of their model and found that $\eta_{M P}$ does not always agree with $\eta_{C A}$ rather $\eta_{M P}>\mathrm{n}$, but approaches $\eta_{C A}$ in the limit $T_{c} \rightarrow T_{h}$. IO asserted that this difference between $\eta_{M P}$ and $\eta_{C A}$ is due to additional heat transfers which may be missed in the original derivation of $\eta_{C A} \cdot{ }^{3}$ Recently, increasing attention has been drawn to optimize heat engines which do not operate in the maximum power regime, instead, under the compromise between the energy benefit and the power 
loss. To evaluate this compromise, ecological criterion $(E=$ $\left.\mathrm{P}-\mathrm{T}_{0} \sigma\right)^{4,55}$ and a unified optimized $\Omega$ criterion $\left[\Omega=\left(2 \eta-\eta_{C}\right)\right.$ $P / \eta]^{7}$ have been proposed to optimize real heat engines, where $P$ is the power output $T_{0}$ is the environmental temperature, and $\sigma$ is the entropy production rate. Further, it was proved that the $\Omega$ function is equivalent to the $E$ function (uniformly called $E-\Omega$ function)..$^{56}$ The important feature of the maximum $\Omega$ criterion is that it gives an optimized efficiency lying between the maximum efficiency and the EMP, i.e., $\eta_{C A}<\eta_{m \Omega}<\eta_{C^{\circ}}$ Angulo-Brown et al. ${ }^{57-58}$ first discussed about the possibility of thermodynamic optimization in some biochemical reactions within FTT frame work. The expansion of the optimization efficiency (near equilibrium) $\eta_{E \Omega}=3 \eta_{c} / 4+\eta_{c}^{2} / 32+O\left(\eta_{c}{ }^{3}\right)$, has also been proved from the symmetry of the Onsager coefficients, where the coefficient 3/4 is universal for the strong coupling condition in the linear response regime. ${ }^{56,59}$ Further, it has been shown that the quadratic coefficient $1 / 32$ is also universal for the strongly coupling systems with the left right symmetry. ${ }^{56}$ The universality of such an efficiency has been investigated in different heat engines such as stochastic Brownian heat engines, ${ }^{60}$ Feynman ratchet heat engines, ${ }^{60}$ quantum dot heat engines, ${ }^{60}$ low-dissipation heat engines, ${ }^{61}$ classical heat engines, ${ }^{62}$ and minimally nonlinear irreversible heat engines ${ }^{63}$ and laser quantum heat engine ${ }^{64}$ Another optimization criterion is Efficient power, defined as the product of efficiency and power $(\eta P)$, pays equal attention to both the efficiency and the poweroutput, was first proposed in Refs. $[65,66]$ and further extended in studies. ${ }^{12-13,67-69}$

This review article will report the advances in the efficiency derivation of different models of heat engines. The paper is organized as follows. In Section 2, we discuss about the Maximum power criterion and its applications to different models of engines such as Endoreversible model, stochastic model, low dissipation model etc... Section 3 will be devoted to the description of maximum ecological (or Omega criterion) and its applications to the same models. Results of MP and ME criterion will be compared for these models. In section 4, numerical simulations for efficiency calculation under MP and ME criterion will be discussed. Section 5 and Section 6 presents the brief discussion on efficient power and multiparameter objective criterion. Finally, we will discuss and conclude our review in Section 7 and Section 8.

\section{Efficiency at Maximum Power (EMP) or Maximum Power (MP) Criterion}

\section{Curzon Ahlborn Engine or Endoreversible Carnot Cycle}

The pioneer work to optimize the heat engines at maximum power conditions was done by Curzon Ahlborn in $1975^{3}$ keeping in view the practical importance of heat engine.
Let us briefly discuss about Curzon Ahlborn heat engine:

\section{Model}

The T-S diagram of an Endoreversible Carnot cycle is shown in Figure 1. The cycle operatesbetween a heat source of temperature $T_{h}$ and a heat sink of temperature $T_{c}$. The heat engine considered by Curzon and Ahlborn operates in a Carnot-like cycle consisting of the following four processes.

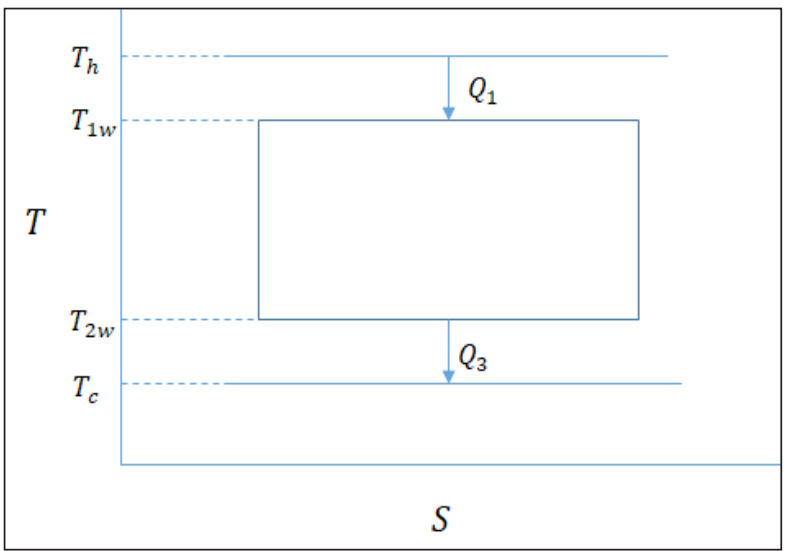

Figure I.Endoreversible Carnot Cycle

Isothermal expansion process: In this process, the working substance absorbs heat, $Q_{1}=\alpha\left(T_{h}-T_{1 w}\right) t_{1}$, from the hot reservoir at temperature $T_{h}$ during the time interval of $t_{1}$ and thus expands, where $\alpha$ is heat conductivity. The effective temperature of the working substance is assumed to be $T_{1 w}\left(<T_{h}\right)$, which is a constant. The total entropy production in this process is $\Delta \mathrm{S}_{1}=Q_{1} / T_{h}+\Delta \mathrm{S}_{1}^{i r}$, where $\Delta \mathrm{S}_{1}^{i r}>0$ is the irreversible entropy production. Due to the convenient consideration, we take the Boltzmann factor $k_{B}=1$ in the whole review.

Adiabatic expansion process: In this process, there will be no heat exchange with reservoirs and the entropy change will be zero, i.e., $Q_{2}=0$ and $\Delta S_{2}=0$. Let be the time taken to complete this process.

Isothermal compression process: In this process, the working substance releases heat, $Q_{3}=\beta\left(T_{2 w}-T_{c}\right) t_{3}$, to the cold reservoir at temperature $T_{c}$ and is compressed, where $\beta$ is heat conductivity. The time for completing this process is assumed to be $t_{3}$. The effective temperature of the working substance is assumed to be $T_{2 w}\left(>T_{c}\right)$, which is a constant. The total entropy production in this process is $\Delta \mathrm{S}_{3}=-Q_{3} / T_{c}+\Delta \mathrm{S}_{3}^{i r}$ , where $\Delta \mathrm{S}_{3}^{i r}>0$ is the irreversible entropy production.

Adiabatic compression process: In this process also, both the heat exchange and the variation of entropy are vanishing, i.e., $Q_{4}=0$ and $\Delta S_{4}=0$. After a whole cycle, the working substance regains its initial state. As a slight deviation from the classical cycle analysis, we consider the four processes taking place simultaneously rather than sequentially.

The total time for the cycle is proportional to the time for 
completing the two isothermal processes, i.e., $t_{\text {tot }}=\xi\left(t_{1}+\right.$ $\left.t_{3}\right)$, where $\xi$ is a constant. Since $\Delta S_{1}^{i r}, \Delta S_{3}^{i r}>0$, the engine operating between the two reservoirs at temperatures $T_{h}$ and $T_{c}$ is irreversible. However, as shown in Figure 2, the irreversible engine can be mapped onto a reversible engine ${ }^{70}$ working between two reservoirs at effective temperatures $T_{1 w}$ and $T_{2 w}$. This assumption can be expressed as

$Q_{1} / T_{1 w}-Q_{3} / T_{2 w}=0$

So the Curzon-Ahlborn heat engine is also called the Endoreversible engine.

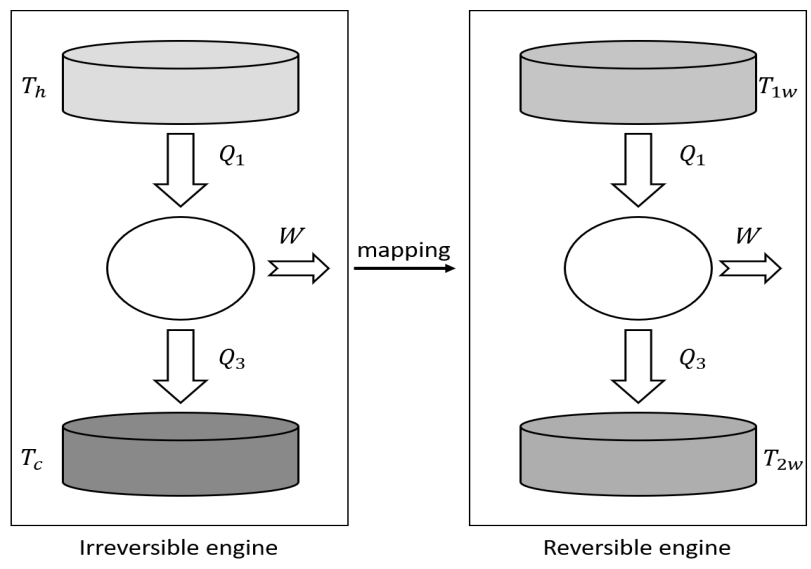

Figure 2.Endoreversible assumption

\section{Optimization}

The net work delivered by the Curzon-Ahlborn heat engine is given as $W=Q_{1}-Q_{3}$. The power output for heat engine is defined as $P=W / t_{\text {tot }}$. To optimize the engine, power is maximized w.r.t $T_{1 w}$ and $T_{2 w}$, i.e., by setting $\partial P / \partial T_{1 w}=\partial P /$ $\partial T_{2 w}=0$ and we obtain

$T_{2 w} / T_{1 w}=\sqrt{T_{c} / T_{h}}$

The efficiency is defined as $n=W / Q_{1}$. Using Eq. (1), we have

$\eta=1-T_{2 w} / T_{1 w}$

By making use of Eq. (2) in above expression, we obtain Curzon Ahlborn (CA) efficiency as

$\eta_{C A}=1-\sqrt{T_{c} / T_{h}}$

This relation has in fact been obtained by Novikov ${ }^{71}$ and Chambadal ${ }^{72}$ when they investigated the efficiency of atomic power stations. For the historic cause, Eq. (4) is still called the Curzon-Ahlborn efficiency in most literature.

\section{Endoreversible Systems with Different Heat Transfer Laws ${ }^{15}$}

The Curzon Ahlborn engine showed the irreversibilities due to finite heat conduction and it directly influenced the behavior of Endoreversible systems. Newtonian heat conduction was chosen to model the heat transfer between engine and thermal reservoirs. In general, these heat transfer laws can be much complicated and thus drastically influence the performance of Endoreversible heat engines. Let us discuss some different heat transfer law used in literature in context with Curzon-Ahlborn engine. All these transfer laws will be expressible as the function of temperatures of the two-contact connected.

\section{- Newton Law}

The Newton heat transfer law has already been used in this Ref. ${ }^{3}$ It assumes that the flow of heat is proportional to the temperature difference between two contacts 1 and 2 as $q=K\left(T_{1}-T_{2}\right)$

Heat flux in solids can be well approximated by a linear heat transfer law. Due to its simplicity the Newton heat transfer law is widely used and many authors have studied the performance of Endoreversible engines, coolers and heaters based on such a linear heat transfer law. ${ }^{18,73-77}$

\section{- Fourier Law}

For the Fourier heat transfer law, the heat flux, $q$, is proportional to the difference of the inverse of temperatures of contacts 1 and 2 as

$q=K\left(\frac{1}{T_{2}}-\frac{1}{T_{1}}\right)$,

where $K$ is Onsager coefficient. This type of heat transfer is often found in conjunction with linear irreversible thermodynamics, as there the driving force corresponding to the heat flux is nothing but the difference of the inverse temperatures. The Fourier heat law has been used in endoreversible systems, ${ }^{78}$ like the Curzon Ahlborn model for example. If the Fourier heat transfer law is used in both heat exchangers of the Curzon Ahlborn model or if equations (6) and (7) are replaced with $Q_{1}=\alpha\left(1 / T_{1 W}-1 / T_{h}\right)$ $t_{1}$ and $Q_{3}=\beta\left(1 / T_{c}-1 / T_{2 W}\right) t_{3}$, respectively, through similar calculations, Chen and Yan $^{22}$ derived the EMP to be

$\eta_{C Y}=\frac{\eta_{c}}{2-\gamma_{C Y} \eta_{c}}$

with $\gamma_{C Y}=1 /(1+\sqrt{ } \beta / \alpha)$.

\section{Radiation}

Hot body like sun emits EM radiation and that too can serve as a source of heat for heat engines. Radiative heat transfer is typically described by the Stefan-Boltzmann law for black body radiation and the heat flux between two radiating bodies attemperature $T_{1}$ and $T_{2}$ is given as

$q=\left(K_{1} T_{1}^{4}-K_{2} T_{2}^{4}\right)$,

The coefficients $K$ are proportional to the Stefan-Boltzmann Constant, the emittance of the two radiating bodies and geometry factors.

\section{Dulong-Petite}

There are some physical situations where the heat transfer between two sub-systems involves conductive as well as radiative components. A combined conductive-convective and radiative heat transfer can be described in a simplified 
fashion and is called Dulong-Petit law as

$q=K\left(T_{1}-T_{2}\right)^{n}$

where $K$ is a proportionality constant. The value of the exponent is usually in the range between 1.1 and 1.6. Angulo-Brown and Paez-Hernandez ${ }^{79}$ investigated the Curzon Ahlborn model using Dulong Petit heat conduction law with $n=5 / 4$ and Chen and Yan et al. ${ }^{80-82}$ also examined the forward and reverse Carnot cycles with Curzon Ahlborn model for arbitrary $n$.

\section{Generalized Heat Transfer Law}

Some authors generalized the above discussed heat transfer laws and analyzed systems obeyinga non-linear heat transfer law of the form

$q=\left(K_{1} T_{1}^{n}-K_{2} T_{2}^{m}\right)$

Which includes the Newton $(n=m=1)$, Fourier $(n=m=-1)$, and radiative $(n=m=4)$ heat transfer laws as special cases. The influence of the non-linear heat transfer law $q=K\left(T_{1}{ }^{n}\right.$ $T_{2}{ }^{n}$ ) on the Curzon-Ahlborn model was studied by Chen ${ }^{22}$, Gordon ${ }^{24}$ and Nulton et al. ${ }^{83}$

\section{Stochastic Heat Engines}

In 2008, Schmiedl and Seifert ${ }^{29}$ constructed a stochastic heat engine by using an optical trap to control a Brownian particle to perform a Carnot-like cycle and thus derived the EMP within the context of stochastic thermodynamics. ${ }^{84-86}$

\section{Model}

The controlled particle performs the following four processes similar to Carnot-cycle:

Isothermal expansion process: During this process, a certain amount of heat $Q_{1}$ is absorbed from the hot reservoir at temperature $T_{h}$ by the particle embedded in it. The governing potential $\mathrm{V}\left(\mathrm{r}, \lambda_{1}(\tau)\right)$ is time-dependently varied by coordinating the intensity of the optical trap during $0<$ $\tau<t_{1}$, where $\lambda_{1}(\tau)$ represents the protocol of coordination. There will be an entropy change during this process.

Adiabatic expansion process: During this process, there is no heat exchange as well as no entropy change i.e $Q_{2}=$ 0 and $\Delta \mathrm{S}_{2}=0$. This is an instantaneous process where the temperature is switched from $T_{h}$ to $T_{c}$ at time $\tau=t_{1}$ The position distribution of the Brownian particle does not change during this step. To keep the distribution unchanged, the potential also needs a corresponding sharp change.

Isothermal compression process: During this process, the particle is embedded in a cold reservoir at temperature $T_{c}$ and a certain amount of heat $Q_{3}$ is released to the cold reservoir. The potential is time-dependently changed during $t_{1}<\tau<t_{1}+t_{3}$. The protocol of coordination is denoted by $\lambda_{3}(\tau)$. Again, in this process, there will be net change in the entropy.
Adiabatic compression process: Similar to the adiabatic expansion process, this is also an instantaneous process and there will be vanishing entropy change and no exchange of heat with the reservoir, i.e., $Q_{4}=0$ and $\Delta \mathrm{S}_{4}=0$. After a whole cycle, the position distribution of the Brownian particle returns to its initial distribution. Thus, the changes of the total energy and the entropy vanish.

The key assumption is that the position distribution $p(r, t)$ of the Brownian particle in an isothermal process at temperature $T$ satisfies the stochastic dynamics as $\partial p(r, \tau) /$ $\partial \tau=-\boldsymbol{\nabla} . \boldsymbol{j}$, where $\boldsymbol{j}$ and $\boldsymbol{\mu} .(\boldsymbol{\nabla} V+\boldsymbol{\nabla}) p(\boldsymbol{r}, \tau)$, is the mobility tensor. Within stochastic thermodynamics frame work, the irreversible entropy production in the isothermal process can be expressed as

$\Delta S^{i r}=T \int_{t_{i}}^{t_{f}} d \tau \times \int d^{3} r \frac{j(r, \lambda(\tau)) \cdot \mu^{-1} \cdot j(r, \lambda(\tau))}{p(r, \tau)}$,

where $\boldsymbol{\mu}^{-1}$ is the inverse of the mobility tensor, $t_{i}$ and $t_{f}$ represent the start and the end time of the process, respectively, $T$ is the temperature which is equal to $T_{h}$ and $T$ for the isothermal expansion process and the isothermal compression process, respectively and $\lambda(\tau)$ represents the protocol and equals $\lambda_{1}(\tau)$ and $\lambda_{3}(\tau)$ for the isothermal expansion process and the isothermal compression process, respectively. For a given protocol, it is observed that minimum irreversible entropy production in the isothermal process has the form

$\min \left\{\Delta S^{i r}\right\}=\frac{A}{T\left(t_{f}-t_{i}\right)}$,

where $A$ is the irreversible action named by Schmeild and Seifert. $^{29}$

\section{Optimization}

Similar to the thermodynamics analysis in CA engine, the expressions of power and efficiency for the stochastic heat engine can be given as

$P=\frac{\left(T_{h}-T_{c}\right) \Delta S-\left(T_{h} \Delta S_{1}^{i r}+T_{c} \Delta S_{3}^{i r}\right)}{t_{1}+t_{3}}$,

$\eta=\frac{\left(T_{h}-T_{c}\right) \Delta S-\left(T_{h} \Delta S_{1}^{i r}+T_{c} \Delta S_{3}^{i r}\right)}{\left(T_{h}\left(\Delta S-\Delta S_{1}^{i r}\right)\right)}$

Power maximization can be done by first minimizing $\Delta \mathrm{S}_{1}$ ir and $\Delta \mathrm{S}_{3}$ ir with respect to the protocols for given time $t_{1}$ and $t_{3}$, which gives the optimized protocols $\lambda_{1}{ }^{*}(\tau)$ and $\lambda_{3}{ }^{*}(\tau)$ After that, power is maximized with respect to time $t_{1}$ and $t_{3}$ for the optimized protocols. With reference to Eq. (12), the minimum irreversible entropy productions in two isothermal processes can be expressed as $\min \left\{\Delta S_{1}{ }_{1}{ }^{2}\right\}=A_{1} / t_{1}$ and $\min \left\{\Delta S_{3}{ }^{i r}\right\}=A_{3} / t_{3}$. Substituting them into Eq. (13) and maximizing the power with respect to $t_{1}$ and $t_{3}$, we obtain the optimized time as

$t_{l}^{*}=\frac{2 \sqrt{A_{l}}\left(\sqrt{A_{1}}+\sqrt{A_{3}}\right)}{\left(T_{h}-T_{c}\right) \Delta S},(l=1,3)$,

By substituting the above equation and the minimum irreversible entropy productions (Eq. (12)) into Eq. (14), we can derive the EMP, also called Schmeild and Seifert 
efficiency $\left(\eta_{s s}\right)$, as

$$
\eta_{S S}=\frac{\eta_{C}}{2-\gamma_{S S} \eta^{\prime}}
$$

where $\gamma_{S S}=1 /\left(1+V A_{3} / A_{1}\right)$. In particular, when aharmonic potential is used to represent the effect of the optic trap i.e. $A_{1}=A_{3}$, the above equation is reduced to

$\eta_{S S}^{\prime}=\frac{2 \eta_{C}}{4-\eta_{C}}$

Another model of stochastic heat engine was studied in Ref. [87] in which authors had investigated the stochastic thermodynamics of a two-particle Langevin system. The model consists of two Brownian particles in one dimension which are trapped by a harmonic potential and driven by a linear external force. Two particlesare in contact with a heat bath at different temperatures. This temperature difference induces a heat flow, and thus enabled the system to work against the external force. The system act as an autonomous heat engine performing work against the external driving force. The derivation of EMP for this engine is found to be same as CA efficiency which is the universal result of Endoreversible heat engines but this engine operates in non-equilibrium condition and thus not Endoreversible.

\section{Feynman's Ratchet as a Heat Engine}

To explain the second law of thermodynamics, Feynman introduced an imaginary microscopic ratchet device in his famous lectures. ${ }^{88}$ Feynman's ratchet, as a parental model, was investigated by many researchers.$^{89-92} \mathrm{~A}$ related study of interest is efficiency at maximum power of Feynman ratchet since the processes of heat and work transfer are assumed to occur at finite rates, thus generating a finite output power.In particular, EMP of Feynman's ratchet was obtained by optimizing the external load for a given internal parameter in Refs. [90-92] which was investigated further by optimizing both the internal parameter and the external load of the ratchet device. ${ }^{30}$ The main ideas and findings of the work for EMP on this system will be discussed here. ${ }^{30}$ The ratchet is shown below in Figure 3.

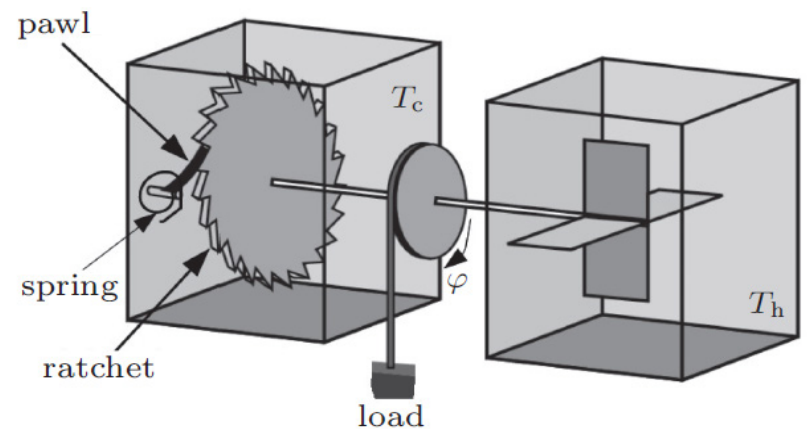

Figure 3.Feynman's Ratchet Device

$R_{F}=r_{0} e^{-\frac{(\epsilon+M \theta)}{T_{h}}}$,

where $r_{0}$ is a rate constant with units of $s^{-1}$. A part of this heat is converted into work $M \theta$, and the remaining energy is eventually transferred as heat $\epsilon$ to the cold reservoir through the interaction between the ratchet and the pawl. Similarly, in the backward step, energy $\epsilon$ should be accumulated from the cold reservoir to lift the pawl high enough so that the ratchet can slip. The rate to get this energy is

$R_{B}=r_{0} e^{-\frac{\epsilon}{T_{C}}}$

In the backward process, the work done by the load is $Z \theta$. This energy and the accumulated energy $\epsilon$ are returned to the hot reservoir in the form of heat. Using the rates of forward and backward rotations, the power of Feynman's ratchet system is given as

$$
\begin{aligned}
P & =\left(R_{F}-R_{B}\right) M \theta \\
& =r_{0} M \theta\left[e^{-\frac{(\epsilon+M \theta)}{T_{h}}}-e^{-\frac{\epsilon}{T_{C}}}\right]
\end{aligned}
$$

By assuming that the heat leakage due to the kinetic energy vanishes (perfect ratchet device), the net rate of heat absorption from the hot bath via the potential energy $y^{93-94}$ may be expressed as:

$\dot{Q}_{1}=\left(R_{F}-R_{B}\right)(\epsilon+M \theta)$

Therefore, the efficiency can be expressed as

$\eta=\frac{P}{\dot{Q}_{1}}=\frac{M \theta}{\epsilon+M \theta}$

It is to be noted that $P$ depends on the internal parameter $\epsilon$ and the external load $Z$. It is easy to tune the external load $Z$. Here, both $\epsilon$ and $Z$ are optimized to achieve the maximum power. After maximizing power, we obtain optimized values $\epsilon^{*}$ and $Z^{*}$, which in turn, substituted in Eq. (22) to obtain efficiency at maximum power $\left(\eta_{T}\right)$. The EMP for Feynman's ratchet device is

$\eta_{T}=\frac{\eta_{c}^{2}}{\eta_{c}-\left(1-\eta_{c}\right) \ln \left(1-\eta_{c}\right)}$

It is observed that above result is slightly higher than obtained $\eta_{C A}$ by Curzon and Ahlborn for macroscopic heat engines and $\eta_{S S}$ obtained by Schmiedl and Seifert for stochastic heat engines. Efficiency at maximum power of Feynmann ratchet have been studied further as hot and cold ratchet with numerical examples, ${ }^{95}$ by using thermoelectric transport theory ${ }^{96}$ etc. The Feynman-Smoluchowski (FS) ratchet $^{88,97}$ is studied in the high temperature regime and thus universality of EMP (see section below) up to second order is reproduced through non-linear approximation in the output power. ${ }^{98}$

\section{Universality of EMP}

One of the most profound findings is the universality of the EMP ${ }^{32}$ and Van den Broeck, Esposito and Lindenberg had done remarkable contribution to study the universal behaviour of EMP. Firstly, Van den Broeck ${ }^{26}$ proved that, in linear regime, more precisely to linear order in $\eta_{C^{\prime}}$ EMP is indeed limited by the CA efficiency, and is exactly half of the Carnot efficiency, as $\eta \leq \eta_{C A}=\eta_{c} / 2+O\left(\eta_{c}^{2}\right)$. The upper 
limit is reached for a specific class of models, namely, those for which the heat flux is strongly coupled to the workgenerating flux. Later, in 2009, Esposito et al. ${ }^{32}$ constructed a general heat engine and verified that the EMP exhibits universality up to a quadratic order term of $\eta_{c}$ for the strong coupling system in the presence of a left-right symmetry. Thus, when EMP is investigated at small relative temperature difference (or near equilibrium), it has been observed that EMP in all cases yield similar terms up to second order as

$\eta_{M P}=\eta_{c} / 2+\eta_{c}^{2} / 8+O\left(\eta_{c}^{3}\right)$,

where the linear coefficient $1 / 2$ is universal for the systems operating under the strong coupling condition in the linear response regime. Beyond the linear response, the universal value of the quadratic coefficient is equal to $1 / 8$ for the strong coupling systems in the presence of left-right symmetry. ${ }^{32}$ The above universality predictions have been confirmed in a number of heat engine systems involving classical and quantum regimes. ${ }^{33-34,99-102}$

\section{EMP Bounds and Low Dissipation Heat Engines}

In Endoreversible models, ${ }^{3,103-104}$ the working medium is assumed to be internally reversible and also there are no heat leaks between the heat baths. The source of irreversibility is solely due to the finite rate of heat transfer between the working medium and the external thermal baths. However, CA efficiency is not a universal result, and also it is neither an upper nor a lower bound. Recently, heat engines have been studied under another type of expansion, namely the so-called low-dissipation limit, ${ }^{34}$ which departs from a first order approximation in the entropy generation of irreversible heat devices. In this limit, not the thermodynamic forces, but rather the operation times for the various stages of the engine are the central quantities. It is assumed that reversible operation is attained when these times go to infinity. Maximum power is achieved by optimization of the operation times. It was shown that the Curzon-Ahlborn efficiency is reproduced for a specific optimization procedure of this type. The authors in Ref. [105] presents a general thermodynamic frame work for a work producing engine, which cyclically runs through a number of stages $\mathrm{j}=1, \ldots, \mathrm{N}$ such that the system can be in contact with ideal reservoirs of heat and particles (including in particular an adiabatic transformation with no exchange of energy or particles) during these stages. In accordance with the second law, the entropy change in a system is (in each step of the cycle) the sum of the entropy exchange with the reservoir and a non-negative entropy production term given as

$\Delta S_{j}=\Delta_{e} S_{j}+\Delta_{i} S_{j}$

where $\Delta_{e} S_{j}=\frac{Q_{j}}{T_{j}}$,

and $\Delta_{i} S_{j} \geq 0$
Let the durations of each stage $j$ is $\tau_{j}$. In the limit $\tau_{j} \rightarrow \infty$ $\forall j$, the corresponding infinitely slow process becomes reversible, hence $\Delta S_{j} \rightarrow 0, \forall j$. By operating at a finite time, but still close to the reversible limit, dissipation increases as the inverse of these operation times and is called weak dissipation or Low dissipation (LD) limit, i.e.,

$T_{j} \Delta_{i} S_{j}=\frac{\sigma_{j}}{\tau_{j}}+O\left(1 / \tau^{2}\right)$

This type of dependence has been observed in a number of explicit model calculations. ${ }^{37-39}$ It also appears as a lower bound in more general discussions. ${ }^{106-107}$ The assumption is expected to be valid if the set-up (system plus contact to reservoirs) has a smallest non vanishing relaxation time. The parameters $\sigma_{j}$ incorporate information such as system and contact characteristics and operational prescription. All irreversibilities (dissipations) are incorporated in the parameters, which play the same role as the thermal conductances of the CA-model. This framework can be used to investigate various types of thermodynamic machines. In Ref. [34] authors consider a minimal and generic model of a standard Carnot engine, operating between a hot and a cold reservoir at temperature $T_{h}$ and $\mathrm{T}_{\mathrm{c}}\left(<\mathrm{T}_{\mathrm{h}}\right)$. We will refer to the corresponding stages as $j=h$ and $j=c$. The other stages are adiabatic, i.e., $\Delta_{e} S_{j}=0$, for $j \neq h$ and $j \neq c$. Power is maximized as a function of the operation times i.e. $\tau_{j}$ and then solving the equation $\frac{\partial P}{\partial \tau_{j}}=0, \forall j$ for operational times and then efficiency at maximum power is then calculated as

$\eta^{*}=\frac{\eta_{C}\left(1+\sqrt{\frac{\sigma_{h}}{\sigma_{c}}}\right)}{2+\left(2-\eta_{c}\right) \frac{\sigma_{h}}{\sigma_{c}}}$

For "symmetric dissipation", more precisely $\sigma_{c}$ $\sigma_{h}=1$ one recovers the Curzon-Ahlborn efficiency $\eta^{*}=\eta_{C A}=1-\sqrt{1-\eta_{C}}$. Further, the limits $\sigma_{h} / \sigma_{c} \rightarrow 0$ and $\sigma / \sigma_{h} \rightarrow 0$ provide a lower and upper bound, respectively for the efficiency as:

$\frac{\eta_{C}}{2} \leq \eta^{*} \leq \frac{\eta_{C}}{2-\eta_{C}}$

The above analysis includes the simpler case of an Endoreversible 4-stage engine, presented in Ref.[34] These results are consistent with those obtained by Chen and $\mathrm{Yan}^{22}$ based on the Endoreversible assumption and those obtained by Schmiedl and Seifert ${ }^{29}$ for stochastic heat engines which in fact also satisfy the low-dissipation assumption. The important result of above study is that EMP is exactly the CA-value when these constant (dissipation constants) are equal. The $C A$ efficiency is reproduced without invoking any specific heat transfer law. So, one can say that taking the equality of the irreversibility constants as a symmetry condition play the same role as the left-right symmetry of the fluxes in the strong coupling systems. Thus, universality of efficiency at maximum power (up to second order) emerge as a general property linked to symmetric conditions. The authors have generalized the above scenario for a multiple bath heat engine which means 
an engine exchanging heat with more than two baths. The analogue of the Carnot efficiency (reversible efficiency) as the upper bound for this efficiency, will be denoted by $\eta_{\text {rev }}$ The analogue of the Eq. (28) which describe the bounds for a heat engine with multiple baths in low dissipation limit can be obtained as

$\frac{\eta_{\text {rev }}}{2} \leq \eta^{*} \leq \frac{\eta_{\text {rev }}}{2-\eta_{\text {rev }}}$

Thus, low-dissipation limit should generically describe correctly - at least to first order - the irreversible correction to the reversible limit. The endo-reversible approximation on the other hand is a system-dependent approximation, based on the assumption that the dissipation can be neglected in some of the stages (e.g., the adiabatic phases), which may or may not be true.

Wang et al. ${ }^{37}$ showed that the maximum power output corresponds to minimizing the irreversible entropy production in two "isothermal" processes in the Carnot-like cycle. They argued that the low-dissipation assumption is reasonable in the long-time limit, because the "isothermal" process is quasi-static in the long-time limit, and the irreversible entropy production should be vanishing. However, it must be convergent in the short-time limit. Thus, it is necessary to correct the assumption for finite time. The authors emphasized mainly on the assumption that the rate of irreversible entropy production in an "isothermal" process is a quadratic form of heat exchange rate between the working substance and the reservoir and thus minimum entropy production for a given time is given as

$\min \left\{\Delta S^{i r}\right\}=\left\{\begin{array}{c}\frac{\left(T_{l} \Delta S\right)^{2}}{\kappa_{l} t_{l}},\left(t_{l} \rightarrow \infty\right) \\ \Delta S,\left(t_{l} \rightarrow 0\right),(l=\mathrm{h}, \mathrm{c})\end{array}\right.$

where $\kappa_{l}$ can be regarded as the thermal conductivity. Although this assumption is different from the Endoreversible assumption or low-dissipation assumption, but still Eq. (28) is true for this assumption as well. Thus, the low-dissipation assumption is a sufficient condition but not necessary condition for the existence of two bounds $\eta_{c} / 2$ and $\eta_{c} /\left(2-\eta_{C}\right)$.

A minimal nonlinear irreversible model of heat engine was proposed in Ref.[38] which is described by the extended Onsager relations, where a new nonlinear term which corresponds to power dissipation is added to the heat flux from the hot reservoir in the standard Onsager relation and no other nonlinear terms are assumed. The proposed model is a natural and minimal extension of the linear irreversible heat engine. The efficiency at maximum power for this model is upper bounded by $\eta_{C} /\left(2-\eta_{c}\right)$. To demonstrate the validity of above assumption, it has been shown that the finite-time Carnot cycle model, called the low-dissipation Carnot engine, can be described by the extended Onsager relations. The relation between the low-dissipation models and the minimally nonlinear irreversible models under the symmetric dissipation condition was further investigated in Ref. ${ }^{63}$ Later, based on some improvements of the above models, ${ }^{108-114}$ many researchers had derived the useful results for the general expression of EMP and its bounds.

\section{Quantum Dot as a Heat Engine and Non-universality of EMP}

Quantum dots (QDs) are of significant importance because they are ideally the objects with zerodimension and with discrete electronic states, and thus can be used as perfect energy filters which only allow electron transport at a single energy. Because of their potential use in high efficiency devices, the performance of QD heat engines has been studied extensively by theorists. ${ }^{31,33,115-120}$ QD heat engine consists of a single level quantum dot, with orbital energy $\epsilon$, and it exchanges electrons with a cold left lead at temperature $T$, and chemical potential $\mu_{\nu}$ and with a hot right lead at temperature $T_{r}$ and chemical potential $\mu_{r}$ (Figure 4). The quantum dot is either empty (state 1) or filled (state 2). The exchange of electrons between the leads through the dot will be described by a stochastic master equation, ${ }^{121-123}$ and the corresponding thermodynamic properties can be obtained from stochastic thermodynamics. When operating close to equilibrium, Carnot efficiency will be achieved, while Curzon-Ahlborn efficiency will be reproduced at maximum power conditions in the linear regime. In particular, the efficiency at maximum power will be found to be $\eta=\eta_{c} / 2+\eta_{c}{ }^{2} / 8+.$. with the coefficient of $\eta_{c}{ }^{2}$, again equals to $1 / 8$. This provides further support for the thesis of universality for this value, especially since the regime of maximum power is found to lie entirely in the quantum regime. The expansion also features the expected coefficient $1 / 2$ for the linear term.

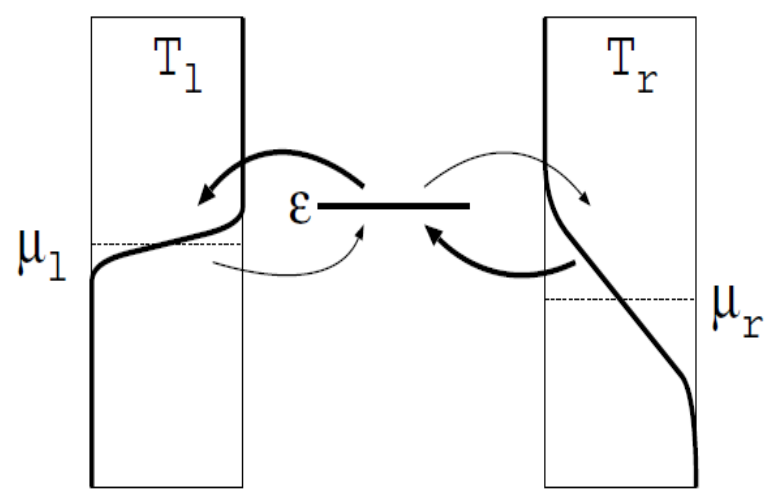

Figure 4.Quantum dot engine

However, it has been observed that this universality may break down for the quantum dot heat engine depending on the constraints imposed, though the tight-coupling condition remains applicable in the sense that the heat flux is directly proportional to the work-generating flux. ${ }^{118}$ When the energy of quantum dot relative to one of the lead's chemical potential is fixed and the other is varied, the $1 / 2$-universality is observed with non-universal second- 
order coefficient depending on the value of the fixed chemical potential, as expected. On the other hand, when the energy of quantum dot is varied with fixed chemical potentials of both leads, we observe a violation of the robust $1 / 2$ universality in near equilibrium expansion of EMP such that the linear coefficient turns out to be unity. Thus, EMP will be much higher compared to the conventional cases. Thus, universality of efficiency requires an additional constraint besides the tight-coupling condition, which turns out to be the applicability of the linear irreversible thermodynamics. $26,38,96,124-127$

\section{Efficiency at some Arbitrary Power}

In recent years, the universality of EMP has been studied extensively in the literature. However, in actual practice, the heat engines may not work in the maximum power regime but rather in the regime with slightly smaller power than the maximum power, yet at a larger efficiency than the EMP. Therefore, it is of great importance to study the efficiencyof heat engines at arbitrary power output. The first steps inthis direction were performed in Refs. [43$46,49,128]$ In Ref.[47] the minimally nonlinear irreversible heat engines at arbitrary power are studied and the lower and upper efficiency bounds under the tight coupling condition for different operating regions have been deduced. In the region of higher loads, a small power loss away from the maximum power results a significant large gain in efficiency. Hence, to achieve higher efficiency, it is advisable to operate the heat engine in a regime, which is a slight deviation from the maximum power regime. The study of thermoelectric quantum heat engines under arbitrary power are presented in Refs. ${ }^{43,128}$ The upper bound of efficiency equals to the Carnot efficiency at zero power output but decays with increasing power output. By using approach of linear irreversible thermodynamics in Ref.[48] universal upper bound of efficiency of steady state heat engines working at arbitrary power has been derived. This study also highlighted that a slight deviationfrom the maximum power conditions can result in higher efficiency, thus making engines more economical.In this context, we recall that any regime in which the efficiency is greater than the efficiency at maximum power and the power is greater than the power at maximum efficiency is considered as an optimum operating regime in FTT.

\section{Maximum Ecological (ME)/ Maximum Omega $(\mathrm{M} \Omega)$ Criterion}

Besides MP Criterion, used as the criterion of merit for the best performance of realistic heat engines, it is possible to use other optimization criterion that makes the best compromise between' power output and "lost power." This criterion has a long-range purpose in the case of being consistent with ecological targets while MP criterion has been recognized for short term goals ${ }^{129}$ as the results showed that ${ }^{4}$ the use of the MP criterion brings about a high-entropy production. Thus, Angulo-Brown had suggested an "ecological" criterion ${ }^{4}$ for the best mode of action of CA type engine which was further improved by Yan. ${ }^{55}$ It consists of optimizing the function $E=P-T_{c} \sigma$, which represents the best compromise between power $P$ and the rate of entropy production $\sigma$ and the temperature of the cold reservoir $T_{c}$, and $T \sigma$ is named "power loss." The ME criterion provides approximately $80 \%$ of the maximum power, but with entropy production of just $30 \%$ of the entropy produced by the MP criterion. In general, ecological function is defined $a s^{55}$

$E=P-T_{0} \sigma$,

where $T_{0}$ is the environment temperature. For a CA engine operating between two reservoirs at a high temperature $T_{h^{\prime}}$ and a low temperature $T_{C^{\prime}}$ using Eq.(31) and the procedures similar to that of Ref.[4] we can conclude that the efficiency at maximum $E$ is given by

$\eta_{E}=1-T_{C} / T_{h} \sqrt{\left(T_{h}+T_{o}\right) /\left(T_{c}+T_{o}\right)}$

In the limit $T_{c} \rightarrow T_{o^{\prime}}$ above equation becomes

$\eta_{E}=1-\sqrt{\frac{T_{c} / T_{h}\left(1+T_{c} / T_{h}\right)}{2}}$

Some authors have also suggested entropy production minimization (MEP Criterion). But, Angulo-Brown had successfully showed that the results with ME criterion, when compared with those obtained by minimum entropy production (MEP) criterion $^{129}$ for similar cycle periods, there is a reduced entropy production and in addition, improves power production by about $10 \%$. Also, the efficiency corresponding to $\mathrm{ME}$ criterion will be an average of Carnot and CA efficiencies. In addition, there is a unified optimization function $\Omega=\left(2 \eta-\eta_{\max }\right) P / \eta$ ( $\Omega$ function $)^{7}$, which is defined by considering a compromise between the useful energy and the lost energy, where is the maximum efficiency of a heat engine. It was proved that the $\Omega$ function is equivalent to the $E$ function (uniformly called $E-\Omega$ function) as shown ${ }^{56}$, where the ecological function $E$ can be further rewritten as

$E=P-T_{c} \sigma$

Using $\sigma=\dot{Q} / T_{c}-\dot{Q}_{h} / T_{h^{\prime}} P=\dot{Q}_{h}-\dot{Q}_{c^{\prime}} \eta_{c}=1-T_{c} / T_{h^{\prime}}$, we obtain $E=P-\dot{Q}_{c}-\dot{Q}_{h} \frac{T_{c}}{T_{h}}$

$E=2 P-\eta_{c} \dot{Q_{h}}=\frac{\left(2 \eta-\eta_{c}\right) P}{\eta}$,

$E=\Omega$.

The implementation of this criterion to heat engines only requires the knowledge of power output $P$ and efficiency $\eta$, is independent of environmental parameters and also does not require the explicit evaluation of the entropy generation. These two criteria have a wide range of applications in many fields. ${ }^{130-137}$ Results show that in all 
cases the $E$ - $\Omega$ criterion predicts an optimum operational regime which is intermediate between those arising from maximum useful energy and from maximum efficiency. In other words, any regime in which the efficiency is greater than the efficiency at maximum power and the power is greater than the power at maximum efficiency is considered as an optimum operating regime in FTT. It is found that for the $E$ and $\Omega$ functions described above, the efficiency at optimum conditions has the same form expressible as $\eta_{E \Omega}=1-\sqrt{\frac{\left(1-\eta_{c}\right)\left(2-\eta_{c}\right)}{2}}$

and this optimized efficiency lying between the maximum efficiency and the EMP, i.e., $\eta_{M P}^{C A} \leq \eta_{E \Omega}^{C A} \leq \eta_{C}$ (CA Model).

\section{Universality of Efficiency, Bounds and its Comparison with EMP}

EMP is verified to be universal up to quadratic order in $\eta_{c}$ for the strong coupling systems in the presence of a symmetry condition $^{32}$. It has been observed that the efficiency at unified trade-off function (maximum $\Omega$-criterion)/ME criterion also show universality up to quadratic term in $\eta_{c}^{56}$, i.e. $\eta_{m \Omega}=3 \eta_{c} / 4+\eta_{c}{ }^{2} / 32+O\left(\eta_{c}{ }^{3}\right)$. Zhang et al. had investigated the efficiency of non equilibrium heat engines based on the master equation model of heat engines and verified that the efficiency at $\mathrm{M} \Omega$-criterion/ME criterionexhibits universality up to quadratic order in the deviation from equilibrium for the strong coupling system in the presence of a symmetry condition. This kind of universality is not exclusive of the maximum power regime. It is possible to obtain other performance criteria generating optimized efficiency with the same kind of universality which also behave as upper bounds. This universal behaviour of maximum $\Omega$-efficiency was already being observed in many models of heat engines such as classical heat engines ${ }^{62}$, stochastic Brownian heat engines ${ }^{60}$, Feynman ratchet heat engines ${ }^{60}$, quantum dot heat engines ${ }^{60}$, low-dissipation heat engines ${ }^{61}$, and minimally nonlinear irreversible heat engines ${ }^{109}$, and others. ${ }^{132,134-135,138}$ We consider here the results for some models of heat engines already being discussed for MP regime and their comparison with maximum power efficiency. Firstly, $\Omega$ function is obtained for the considered models of heat engines, and then, the efficiency under maximum $\Omega$ conditions can be obtained, denoted as $\eta_{m \Omega}$.

- For the Endoreversible Curzon-Ahlborn model, the efficiency at maximumis $\Omega$ given by Eq. (36) as $\eta_{m \Omega}^{C A}=1-\sqrt{\left(1-\eta_{c}\right)\left(2-\eta_{c}\right) / 2}$, which can be expanded as $\eta_{m \Omega}^{C A}=\frac{3 \eta_{c}}{4}+\frac{\eta_{c}^{2}}{32}+\frac{3 \eta_{c}^{3}}{128}+O\left(\eta_{c}^{4}\right)$

- For the stochastic heat engine model by Schmiedl and Seifert ${ }^{29}$, the algebra is straight-forward although cumbersome and the result is given by

$\eta_{S S}=\frac{C+D}{E}$ where

$$
\begin{gathered}
C=2\left(1-\eta_{c}\right)\left[\eta_{c}\left(3+\sqrt{4-2 \eta_{c}}\right)-2\left(2+\sqrt{4-2 \eta_{c}}\right)\right], \\
D=8+4 \sqrt{4-\eta_{c}}-\eta_{c}\left(2+\eta_{c}\right), \\
\left.E=2+\sqrt{4-\eta_{c}}\left[2\left(1-\eta_{c}\right)+\left(2+2 \sqrt{4-2 \eta_{c}}\right)-\eta_{c}\right)\right],
\end{gathered}
$$

Which can be expanded as

$\eta_{m \Omega}^{s s}=\frac{3 \eta_{c}}{4}+\frac{\eta_{c}^{2}}{32}+\frac{\eta_{c}^{3}}{64}+O\left(\eta_{c}^{4}\right)$

- For Feynman ratchet and pawl model considered by $\mathrm{Tu}^{30}$, the algebra is also straightforward and we get

$\eta_{m \Omega}^{T}=\frac{\eta_{c}\left(5 \eta_{c}-6\right)}{7 \eta_{c}-8} \approx \frac{3 \eta_{c}}{4}+\frac{\eta_{c}^{2}}{32}+\frac{7 \eta_{c}^{3}}{256}+O\left(\eta_{c}^{4}\right)$

- Similarly, for the nano-thermoelectric engine model reported by Esposito et al. ${ }^{31}$, the efficiency at maximum power $\Omega$ - conditions can be expanded as

$\eta_{m \Omega}^{E L B}=\frac{3 \eta_{c}}{4}+\frac{\eta_{c}^{2}}{32}+\frac{\left(19+\cosh ^{2}\left(a_{o} / 2\right)\right)}{768} \eta_{c}^{3}+O\left(\eta_{c}^{4}\right)$

It can be seen that all four models reproduce the same efficiency up to quadratic order when expanded nearequilibrium (small temperature difference) as coefficients $3 / 4$ and 1/32 appear in all linear and quadratic terms and model-dependent differences can be observed at third and higher terms. The 3/4 coefficient has been also observed for a non-isothermal ${ }^{134}$ and isothermal heat engine ${ }^{59}$ within the linear irreversible approach in the limit of strong coupling when the efficiency is calculated under maximum ecological conditions. The results of the optimized efficiencies $\eta_{m \Omega}$ are plotted in Figure $5^{60}$, which shows that all curves merge together at small temperature differences (as clear from near-equilibrium expansion) while deviations (below or above the $\eta_{m \Omega}^{C A}$-value) are appreciable only for relative large temperature differences. From Figure 5, it is also to be noted that the efficiencies under maximum $\Omega$-function behave in the similar manner as the efficiencies under maximum power for the considered models, thus sharing the same kind of universal behaviour. Also, in each case the maximum $\Omega$-function yields higher efficiencies, closer to the Carnot values. In fact, it is easy to check numerically the exact results of the efficiency at maximum $\Omega$ can be approximated by the semi-sum of the Carnot value and the exact results of the EMP, $\eta_{m \Omega}=\left(\eta_{M P}+\eta_{c}\right) / 2$ (semisum rule). ${ }^{4,59}$

The $\Omega$ function is also applied to the unified low-dissipation (LD) model for heat engines and the corresponding efficiency and its bounds are obtained under general and symmetric conditions. ${ }^{61}$ In LD model, the entropy production during the hot (cold) heat exchange process behaves as $\Sigma_{h} / t_{h}\left(\Sigma_{c} / t_{c}\right)$, where $t_{h}$ and $t_{c}$ denotes the corresponding time durations and $\Sigma_{h}$ and $\Sigma_{c}$ are dissipation coefficients which account for irreversibility details. Here, infinite time limit recovers the reversible case. The MP criterion when applied to LD models, allows recovering the CA efficiency for symmetric dissipation, without assuming any specific heat transfer law or the linear-response regime. The authors also derived the lower and upper bounds for the efficiency at maximum 
power which can be attained under extremely asymmetric dissipation limits. Similarly, $\Omega$ - function is maximized for LD model to obtain lower and upper bounds of efficiency by considering, respectively, the asymmetric limits $\Sigma_{h} / \Sigma_{c}$ $\rightarrow 0$ and $\Sigma_{h} / \Sigma_{c} \rightarrow \infty$ :

$\eta_{\Omega}^{-} \equiv \frac{3 \eta_{C}}{4} \leq \eta_{\Omega} \leq \frac{3-2 \eta_{C}}{4-3 \eta_{C}} \eta_{c} \equiv \eta_{\Omega}^{+}$

Under symmetric dissipation i.e. $\Sigma_{h} / \Sigma_{c} \rightarrow 1$, efficiency obtained is same as obtained by Angulo et al. ${ }^{4,139}$ using the ecological optimization for Endoreversible models and is given as

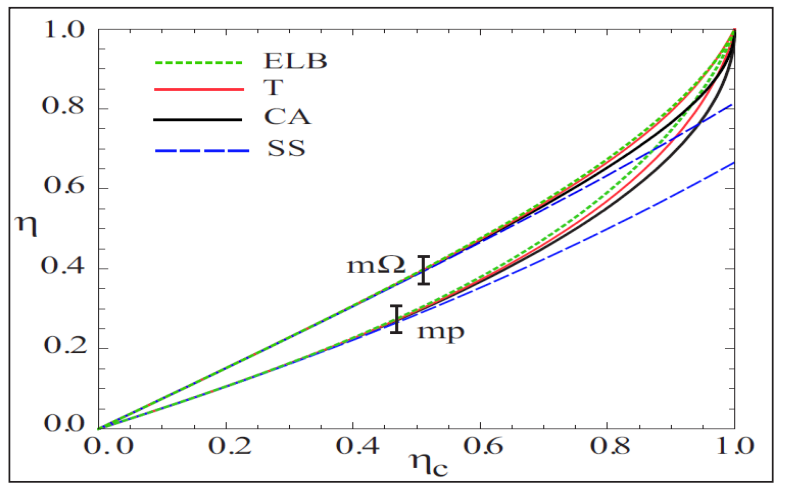

Figure 5.Comparison of efficiency under MP criterion with efficiency under $M \Omega$ criterion as a function of Carnot efficiency for indicated models ${ }^{60}$

$\eta_{\Omega}^{\Sigma_{h}=\Sigma_{c}}=\eta_{\Omega}^{\text {Sym }}=1-\sqrt{\frac{\left(1-\eta_{c}\right)\left(2-\eta_{c}\right)}{2}}$

The $\Omega$-optimization for minimally nonlinear heat engines is conducted in Ref.[140] The results show that under tight-coupling conditions, the efficiency and its bounds in asymmetric dissipation limits are the same as those obtained by de Tomas et al. ${ }^{61}$ for low dissipation heat engines. The efficiency bounds for heat engines under non tight-coupling conditions are also analyzed.

\section{Efficiency Study using Numerical Simulation}

CA efficiency has been validated through many theoretical studies ${ }^{6,26-28,141}$ ranging from the heat engines working under the linear regime $e^{26-28}$ to the heat engines powered through a quantum mechanism, ${ }^{142}$ thus, suggesting a universal nature of CA efficiency. However, to test the validity of CA efficiency experimentally, Y. Izumida et al. ${ }^{50-51}$ performed some numerical experiments by means of Molecular Dynamics (MD) simulations of a weakly interacting gas, which can be treated as a nearly ideal gas, in a finite-time Carnot cycle. The authors studied the efficiency at the MP regime, $\eta_{M P}$ and found that $\eta_{M P}>\eta_{C A}$ but approaches $\eta_{C A}$ in the limit $T_{c} \rightarrow T_{h}$. The assertion given for this difference between $\eta_{M P}$ and $\eta_{C A}$ is the additional heat transfers which may be missed in the original derivation of $\eta_{C A}$. Let us discuss in detail some of the MD simulation experiments performed for different systems to discuss their efficiency under different operating regimes.

\section{Ideal-gas like system}

\section{Model}

This model is originally developed and used by Y. Izumida and $\mathrm{K}$. Okuda. ${ }^{50}$ The model consists of a $\mathrm{N}$ hard disc particles (weakly interacting particles) of diameter $d$ and mass $\mathrm{m}$, confined in a two-dimensional cylinder with rectangular geometry and the collision between hard-disc particles is assumed to be perfectly elastic. The cylinder is fitted with a piston moving back and forth at a constant speed $u$ which is taken to be a control parameter. The system follows four steps to complete a single quasi-static Carnot cycle. The usual quasi-static Carnot cycle of an ideal gas consists of four processes:

a. Isothermal expansion process $\left(V_{1} \rightarrow V_{2}\right)$,

b. Adiabatic expansion process $\left(V_{2} \rightarrow V_{3}\right)$,

c. Isothermal compression process $\left(V_{3} \rightarrow V_{4}\right)$,

d. Adiabatic compression process $\left(V_{4} \rightarrow V_{1}\right)$,

Where $V_{i}^{\prime}$ s are the volumes of the cylinder at which we switch each of the four processes as shown in the Figure 6(a). When we fix $T_{h}, T_{c^{\prime}} V_{1}$, and $V_{2}$, we can easily determine the volumes $V_{3}$, and $V_{4}$ since we assume an ideal gas as the working substance. For an adiabatic quasi-static process with an ideal gas as a working substance, we have the relation $T V^{-1}=$ constant . Here $\gamma$ refers to the ratio of the specific heat capacity at constant pressure to that at constant volume. For a two-dimensional ideal gas, $\gamma$ is 2 . Therefore, $V_{3}=\left(T_{h} / T_{c}\right) V_{2}$ and $V_{4}=\left(T_{h} / T_{c}\right) V_{1}$ for the twodimensional case. In case of finite-time cycle, we assume that the right wall of the cylinder is a piston and moves back and forth at a constant speed $u$. For this model, this $u$ is taken as a unique and controllable parameter. We also assume that each process is switched at the same volume as in the quasi-static case.

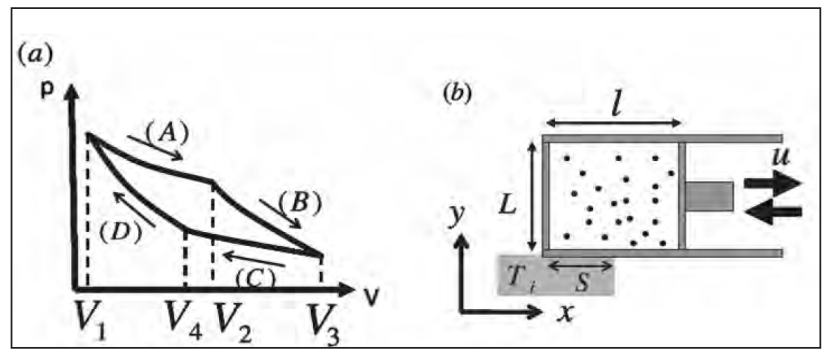

Figure 6.Model description of 2D heat engine Simulation ${ }^{50,143}$

Defining $(x, y)$ coordinates for cylinder shown in Figure 6(b), let the piston move along the $x$-axis at a finite constant speed $u$. Here, the $x$ - length and the $y$ - length of the cylinder are expressed as $/$ and $L$, respectively. Then the volume $V_{i}(i=1, \ldots 4)$ of the cylinder at which each of the four processes are switched (Figure 6(a)) becomes $V_{i}=$ $L I_{i}$, where $I_{i}$ is the $x$-length of the cylinder at the switching 
volume $V_{i}$. If the process $(A)$ begins at time $t=0$, then the volume $V(t)$ of the cylinder at time $t$ is given as

$V(t)=L l(t)=L\left(u t+l_{1}\right)$, for $0<t<\frac{l_{3}-l_{1}}{u}$,

in the expansion processes $(A)$ and $(B)$ and

$V(t)=L\left(-u t+2 l_{3}-l_{1}\right)$ for $\frac{l_{3}-l_{1}}{u}<t<\frac{2\left(l_{3}-l_{1}\right)}{u}$,

in the compression processes $(C)$ and $(D)$.

The collision of a particle with the piston can be classified into two categories:

- Piston moving forward (expansion process) and

- Piston moving backwards (compression process).

\section{Piston Moving Forward}

Denote $v_{o x^{\prime}} v_{f x}$ and $u_{f}$ the initial speed, final speed of the particle and final speed of the moving piston respectively. Conservations of linear momentum and kinetic energy give the relations

$$
\begin{aligned}
& m v_{0 x}+M u=m v_{f x}+M u_{f} \\
& \text { and } \\
& \frac{1}{2} m v_{0 x}^{2}+\frac{1}{2} M u^{2}=\frac{1}{2} m v_{f x}^{2}+\frac{1}{2} M u_{f}^{2},
\end{aligned}
$$

Where $m$ and $M$ are mass of the particle and piston respectively. From these equations, we obtain the relation

$$
v_{f x}=u+u_{f}-v_{0 x}
$$

Since we assume that the mass of the piston is very large compared to the mass of the colliding particle, (i.e $M$ >> $m)$, the speed of the piston does not change appreciably due to collision with a single particle. Therefore, the final speed of a colliding particle with the piston becomes

$v_{f x} \approx 2_{u}-v_{o x}$

The change in kinetic energy $(\Delta K E)$ of a colliding particle, which is the difference between its final and initial kinetic energies, is

$\Delta K E=\frac{1}{2} m\left(v_{f x}^{2}-v_{0 x}^{2}\right)=2 m u\left(u-v_{0 x}\right)$

Since $v_{0 x}$ must be greater than $u$, Eqn. (50) tells us that the colliding particle loses energy, thereby transferring it to the forward moving piston.

\section{Piston Moving Backward}

When the piston is moving backwards (compression process) then two situations arise for the particle collision with the piston. The first and obvious one is when a particle moves to the right. The second is that, while a particle moves to the left, collision occurs as long as the speed of the piston is greater than that the particle. We will see both cases as follows.

If a particle moves to the negative $x$-axis, the laws of momentum and energy conservations can be written as

$-m v_{0 x}-M u=-m v_{f x}-M u_{f}$, and

$$
\frac{1}{2} m v_{o x}^{2}+\frac{1}{2} M u^{2}=\frac{1}{2} m v_{f x}^{2}+\frac{1}{2} M u_{f}^{2}
$$

Again, using the fact that $M>>m$, the final speed of a colliding particle obtained from the above two equations is $v_{f x} \approx-\left(2 u+v_{o x}\right)$

and the corresponding change in the particle's kinetic energy is

$\Delta K E=2 m u\left(u+v_{0 x}\right)$

If a particle moves towards the positive $x$-axis,using the laws of conservation oflinear momentum and kinetic energy, we find the final speed of a colliding particle is

$v_{f x} \approx-\left(v_{0 x}+2 u\right)$,

and the corresponding change in kinetic energy is

$\Delta K E=2 m u\left(u+v_{0 x}\right)$

Thus, it can be concluded from above discussions that, if a particle with the velocity $\overrightarrow{v_{0}}=\left(v_{x}, v_{y}\right)$ collides with the piston whose $x$-velocity is $\pm u$, its velocity changes to $\overrightarrow{v_{f}}=\left(-v_{x} \pm 2 u, v_{y}\right)$. Therefore, the particle does microscopic work of amount $m\left(|\vec{v}|^{2}-\left|\overrightarrow{v_{f}}\right|^{2}\right) / 2=2 m\left( \pm u v_{x}-u^{2}\right)$ against the piston. To simulate the heat reservoirs for isothermal processes, thermal wall is at desired temperature with the length $S$ at the left bottom of the cylinder. The thermal wall has the following feature: ${ }^{144-145}$ The collision of a particle with the thermal wall changes it velocity stochastically to the value governed by the distribution function

$f\left(\vec{v}, T_{i}\right)=\frac{1}{\sqrt{2 \pi}}\left(\frac{m}{k_{B} T_{i}}\right)^{\frac{3}{2}} v_{y} \exp \left(-\frac{m v^{2}}{2 k_{B} T_{i}}\right)$

$\left(-\infty<v_{x}<+\infty, 0<v_{y}<+\infty, T_{i}(i=h\right.$ in $(A), c$ in $\left.(C))\right)$, where $k_{B}$ is Boltzmann constant. The thermal wall may be understood as follows. Imagine a large particle reservoir thermalized at a temperature $T_{i}(i=h$ or $c$ ) instead of the thermal wall and assume that if a particle in the cylinder goes out into the particle reservoir, another particle from the particle reservoir enters into the cylinder. This consideration can be seen as the particle entering in to the cylinder from the particle reservoir obeys the velocity distribution function proportional to the Boltzmann factor multiplied by $v_{y}$ as given in Eq.(57) after normalization. This thermalizing wall guarantees that the particle velocities in the static system are governed by Maxwell-Boltzmann distribution with temperature $T_{i}$ :

$f_{M B}\left(\vec{v}, T_{i}\right)=\frac{m}{2 k_{B} T_{i}} \exp \left(-\frac{m v^{2}}{2 k_{B} T_{i}}\right)$

The heat flowing from the thermalizing wall into the system can microscopically be calculated by the difference of the kinetic energies before and after the collision with the thermal wall. The microscopic heat as well as microscopic work during the simulation can be summed up. At the walls, the reflecting boundary condition for colliding particles are used except for the piston and the thermal wall. 


\section{Simulation Results}

The simulation technique used is event driven molecular dynamics simulation. The values used in Ref.[50] are $N=$ 100 particles with diameter, $d=0.01$ and mass, $m=1$ in the system with $L=1, I_{1}=1, I_{2}=1.5, T_{h}=1, T_{c}=0.7, k_{B}=1$ and length of the reservoir, $S=0.5$. All parameters except $T$ are fixed in all simulation. As time passes, thermodynamic variables should draw a steady cycle independent of initial states. Figure 7, shows the temperature-volume diagram for the steady cycle at $u=0.01$ and $u=0.001$, where $k_{B} T$ is determined by summing up the kinetic energy of all particles and then using the equipartition principle. From this figure, we can see that in the isothermal expansion (compression) process, the temperature approaches a steady value lower (higher) than $T_{h}\left(T_{c}\right)$ at $u=0.01$. This can easily be understood: For a finite $u$, heat should flow into the system at a finite rate to maintain the steady cycle. Therefore, the finite difference of the temperatures between the system and the heat reservoir is necessary. The cycle for $u=0.001$ almost agrees with the quasi-static Carnot cycle of an ideal gas. This implies that considered system of the hard-disc particles closely approximates an ideal gas system. We can see from the figure that as the speed of the piston gets very small, the molecular dynamics simulation result approaches to that of the quasi-static result. This result ensures that the model can describe the ideal gas model heat engine to a good accuracy.

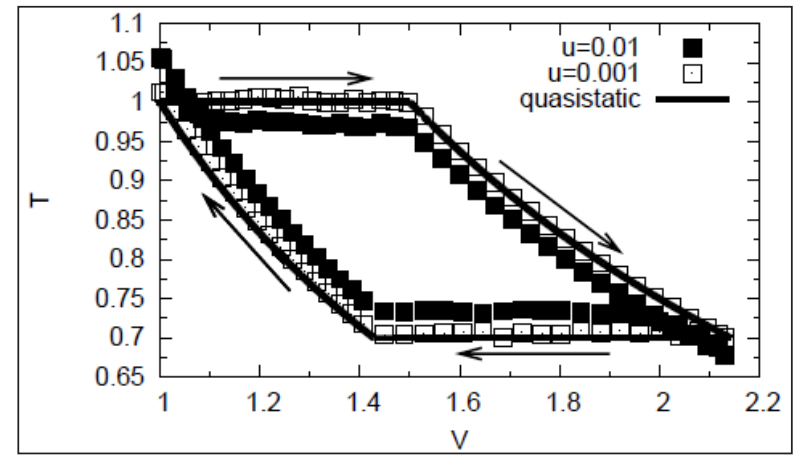

Figure 7.Temperature-volume $(T-V)$ diagram for the steady cycle at $u=0.01$ and $u=0.00 \mathrm{I}$. Solid line is for quasi-static Carnot cycle ${ }^{50}$

The efficiency $\eta=W_{\text {total }} / Q_{h, \text { total }}$ and the power $P=W_{\text {total } / \tau}$ are calculated, where $W_{\text {total }}$ is the total work against the piston, $Q_{h, \text { total }}$ is the total heat flowing into the system from the hot heat reservoir and $\tau$ is the total time for the simulation. Figure 8 , shows variation of power $(P)$ at various $u$. We have found that the maximal power is realized at $u \approx 0.015$.

Figure 9 compares the efficiency at maximal power $\eta_{\max }=$ $\eta\left(u_{\text {max }}\right)$ with the CA efficiency at $T_{h}=1$ and various $T_{c^{\prime}}$ where $u_{\max }$ is the speed giving the maximal power (obtained by plotting power vs piston speed (Figure 8)). We have found that our $\eta_{\max }$ does not always agree with $\eta_{C A}$ but tends to approach $\eta_{C A}$ as $T_{c} \rightarrow T_{h}$ for both of the MD data and the numerical line.

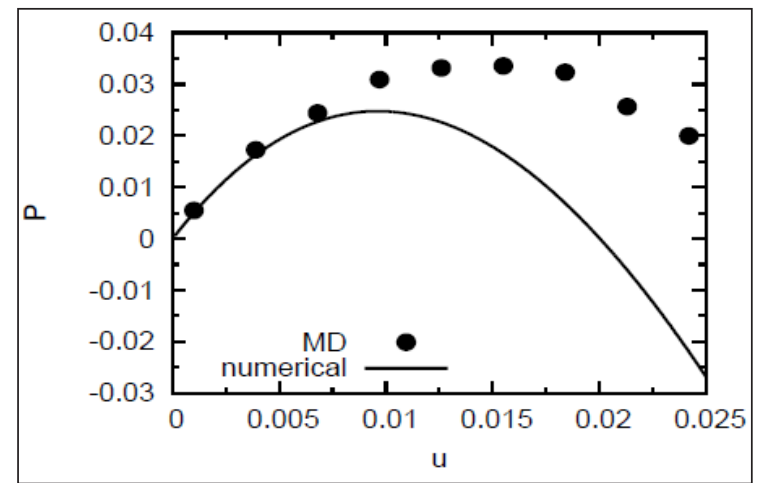

Figure 8.Power $(P)$ vs piston speed $(u)^{50}$

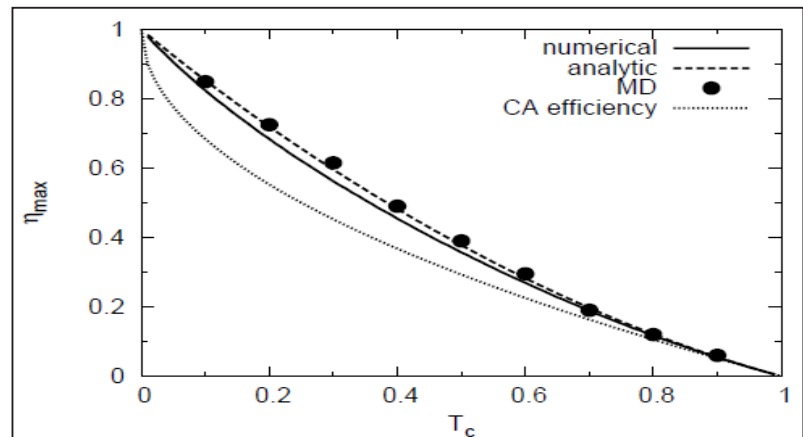

Figure 9. The efficiency at the maximal power $\boldsymbol{n}_{\max } \mathbf{v s} \boldsymbol{T}_{c}^{50}$

The MD simulation efficiencies resulting under MP and ME conditions have been derived and then compared in Ref.[146] by using nearly ideal gas working substance enclosed in a 2D-heat engine. The model is quite similar to that considered by Izumida et al. ${ }^{50}$ Apart from MP and ME criterion, a modified version of function $E^{58,147}$ was taken into consideration for optimization and is given by

$E_{\epsilon}=P(\eta)-\epsilon T_{c} \sigma(\eta)$

Where $\epsilon$ is a parameter that depends on the heat transfer law. If $\epsilon=1$ in Eq. (59), the original ecological function is recovered $^{4}$; on the other hand, when $\epsilon=\sqrt{\tau^{-1}}$, the modified ecological function is obtained for a Newtonian heat transfer law. When $E$ is maximized for a linear heat transfer law the engine efficiency becomes,

$\eta_{E}=1-\sqrt{\frac{(1+\tau) \tau}{2}}=\frac{1}{2}\left(\eta_{c}+\eta_{C A}\right)$,

$\eta_{c}$ being the Carnot efficiency $\eta_{c}=1-\tau$ and $\tau=T_{c} / T_{h}$. On the otherhand, when $E_{\epsilon}$ is maximized for the case of Newtonian heat fluxes, the obtained optimal efficiency is..$^{58,147}$

$\eta_{E_{\epsilon}}=1-\tau^{\frac{3}{4}}$

The MD simulation results under $\mathrm{MP}, \mathrm{ME}$ and $M E_{\epsilon}$ are presented in Figure 10. In the $\tau \rightarrow 1$ limit (where adiabatic processes times are negligible, compared with the isothermal processes times) MD-MP efficiency approaches $\eta_{C A^{\prime}}$, however, for any value of $\tau, M D-M E$ and $M D-M E_{\epsilon}$ 
efficiencies are in good agreement with $\eta_{E \epsilon^{\prime}}$ which is also close to the upper bound given by the low dissipation model with ME criterion $\left(\eta_{M D, L D}^{\text {upper }}\right)$ (see Eq. 42). The shaded region represents the so-called operability region for heat engines. In the operability region, it is observed that as $\tau$ decreases the MD-MP points start approaching $\eta_{E \epsilon}$ and below $\tau=0.5$, efficiency in both cases is better represented by $\eta_{E \epsilon}$ than by $\eta_{C A}$. Thus, interestingly, there is an overall agreement between the computed MD efficiencies at these optimization regimes and $\eta_{E_{\epsilon}}=1-\tau^{\frac{3}{4}}$.

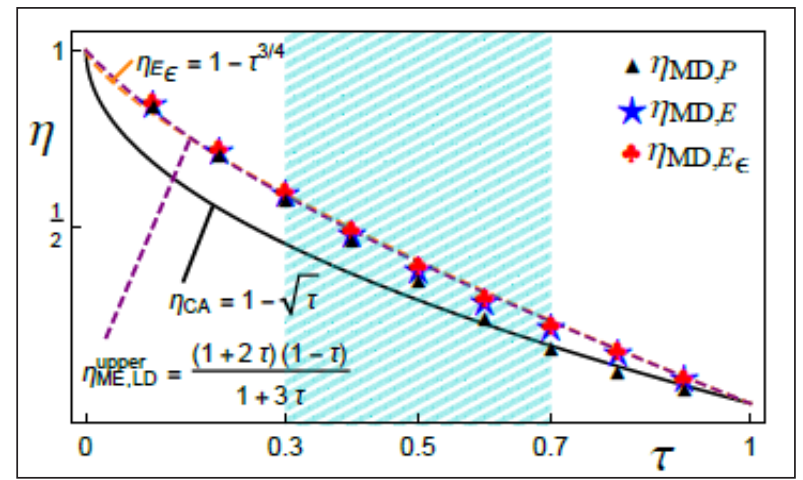

Figure I0.MD simulation results for $M P, M E$ and $M_{E \epsilon}{ }^{146}$ Real-gas like system: ${ }^{148}$

To simulate a real gas in a finite-time Carnot cycle by means of MD simulations, the working substance is chosen to be made of highly dense spherical particles, that is, for which intermolecular interactions are not negligible. The simulation model used in the present work shares some similarities with the approach used by Izumida et al. ${ }^{50}$ To integrate Newton's equations of motion, Velocity Ver let algorithm was used. The efficiency and power of the model heat engine were computed by varying the values of the parameters. In particular, the efficiency at various process rates were compared to theoretical predictions.

\section{Simulation Model}

The model engine consists of a 3-dimensional cubic box of length $L$ containing $N$ identical gas particles of mass $m$. One of the box sides is made of a moveable piston where as the other opposite side is a thermalizing wall, as shown in Figure 6. At each side of the box, periodic boundary conditions are applied on the gas particles, except those at which the piston and thermalizing wall are located. The potential through which the gas particles interact is considered to be Lennard-Jones potential which is given by:

$u^{L J}(r)=4 \varepsilon\left[\left(\frac{\sigma}{r}\right)^{12}-\left(\frac{\sigma}{r}\right)^{6}\right]$

where $r$ is the inter-particle separation, $\varepsilon$ is the depth of the potential well and $\sigma$ is the distance between two particles at which inter-particle potential becomes zero. Moreover, the potential is truncated at a distance $2.5 \sigma$ and shifted, making long range interactions negligible.
Since the potential described by Eq. (62), depends only on inter particle separation, the particles are assumed to be spherical.

\section{Simulation Result}

The values used are $\mathrm{N}=4000, T_{c}=1.25, \mathrm{~L}=50, k_{B}=1, \varepsilon=1$, $\sigma=1$ and $m=1$. All these parameters except the number of particles are in reduced unit and are kept constant for all simulations. The Lennard-Jones parameters $\sigma, \varepsilon$ and $m$ are chosen to be the units of distance, energy and mass respectively. Moreover, the temperature of the cold reservoir becomes $T_{c}=268 \mathrm{~K}$ while the range of variation of the hot reservoir temperature is [310 K-375 K]. As for the piston speed, the range of values studied in the present work is $1.164 \times 10^{-3} \mathrm{~m} / \mathrm{s}$ to $1.164 \times 10^{-1} \mathrm{~m} / \mathrm{s}$. Xenon gas is used for illustration because as a monatomic gas, it is well suited for being modelled by Lenard-Jones spheres The molar volume of the present model system is 0.895 lt/ mol which is small compared to that of an ideal gas, 22.414 $\mathrm{It} / \mathrm{mol}$, but large enough compared to that of water, 0.018 $\mathrm{It} / \mathrm{mol}$. Although it seems that gas is more than 10 times denser than the ideal gas at standard conditions, but there is another method to verify the real gas regime by simply calculating the average inter-particle distance which is about $2.15 \sigma$. Since this value is smaller than the particleparticle interaction cut-off distance $r_{c^{\prime}}$, it implies that each particle is in average within the interaction range of its nearest neighbours. Hence, it is believed the substance is sufficiently concentrated to be treated as a real gas. The engine operates at different piston speeds ranging from $u$ $=0.001$ to $u=0.1$ in the isothermal branches of the cycle, while the adiabatic processes are performed at a uniform speed $u^{\prime}=0.01$ when $u \leq 0.01$ and $u^{\prime}=0.1$ when $u>0.01$. Note that values of $u^{\prime}$ essentially have been set for practical purpose because, it cannot be externally imposed, being the result of the spontaneous expansion (compression) of the substance. It should also be pointed out that another choice for the value of $u^{\prime}$ does not change the overall result as long as $u^{\prime}>u$.

The temperature-volume ( $T-V)$ diagram of finite-time processes for $T_{h}=1.45$ is shown in Figure 11, and for three values of the piston speed including the quasi-static case for comparison purpose. It has been observed that for relatively small piston speeds, the cycle obtained is quite similar to the for the quasi-static process, where as larger piston speed causes a significant departure and thus characterized by a reduction of the area inside the cycle. In fact, the temperature of the gas is not only subjected to fluctuations but its average is systematically higher (lower) to that of the cold (hot) reservoir, the deviation becomes more important as the piston speed increases. This result can be explained with the fact that when a heat engine operates at a finite piston speed, it exchanges heat with 
reservoirs at a finite rate in order to keep a steady cycle. A temperature gradient between the working substance and the heat reservoirs is therefore required to create such conditions.

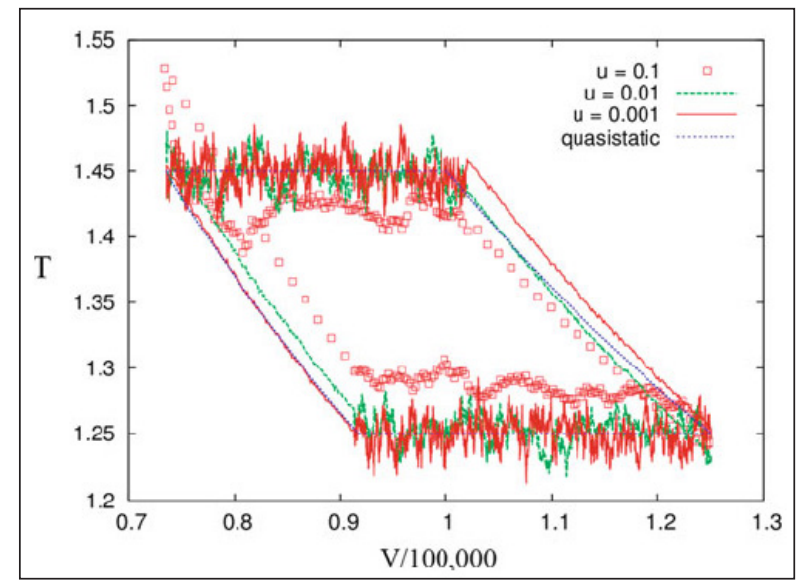

Figure I I.Tempaerature-Volume (T-V) diagram for different pistons speeds ${ }^{148}$

Figure 12 represents the output power per cycle as a function of piston speed for various values of the hot reservoir temperature. The behaviour of all curves is almost similar, that is, power increases initially up to a given value of the piston speed followed by a steady decrease beyond that point. This can be explained as: for slower processes the time needed to complete a full cycle, is too large making the power vanishingly small; on the other hand, for processes at large piston speed, only a small amount of the supplied heat is converted to work, hence, yielding again negligibly small values for $P$. Note that the piston speed at which the power becomes maximum is almost the same for all temperatures $T_{h}$. This interesting finding may indicate that there is an optimum piston speed not dependent upon the temperature of the heat reservoirs, at least for the range of temperatures investigated in the present work and thus may have practical implications on the design of heat engines.

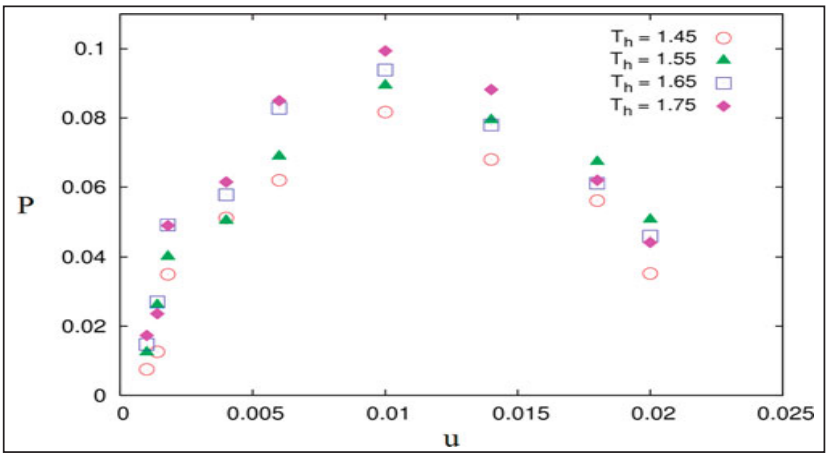

Figure I2.Power dependence on u for different $T_{h}{ }^{148}$

The variation of the efficiency with the temperature ratio $T_{d}$ $T_{h}$ for different values of the piston speed is demonstrated in Figure 13. For all values of piston speeds, the efficiency is monotonically decreasing and approaches zero as the temperature ratio approaches 1 , an expected result. It is clear from the Figure 13 that the efficiency varies linearly with the temperature ratio for a small value of piston speed and this is similar to the quasi-static case for which $\eta_{C} \propto-\frac{T_{c}}{T_{h}}$. For a piston speed close to the one yielding the maximum power (Figure 12), in this case $u=0.01$, the efficiency dependence becomes $1-\left(\frac{T_{c}}{T_{h}}\right)^{a}$, where a $\approx$ 0.43 , which is slightly smaller than the exponent in the expression of the Curzon-Ahlborn efficiency. This implies that the Curzon-Ahlborn efficiency is not well suited to represent model engine based on a highly dense real gas. The operation of such engine can be optimized by applying a unified optimization criterion developed by Hernandez and his colleagues ${ }^{7}$ that makes a good compromise between efficiency and power. The investigation carried out by varying the temperature of the hot heat reservoir, shows that the optimum piston speed depends weakly on temperature.

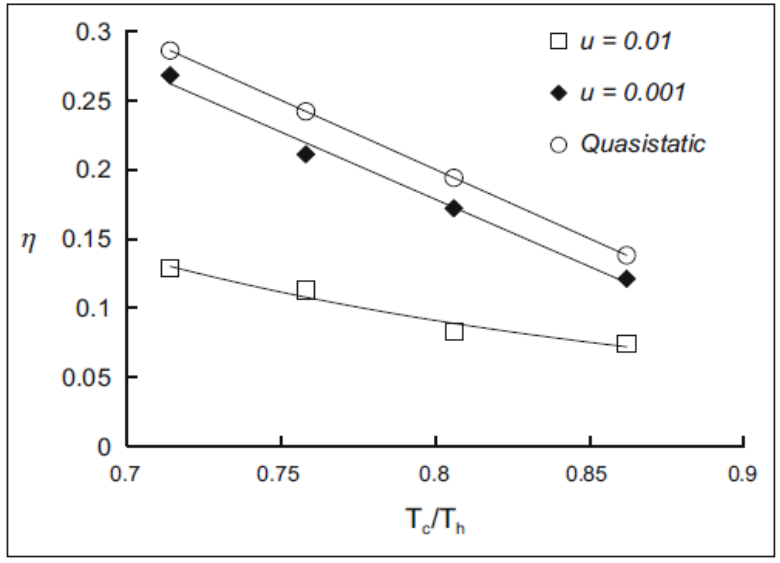

Figure I3.Efficiency dependence on temperature ration for three values of piston speed ${ }^{148}$

\section{Efficient Power Criterion}

Different optimization criteria based on thermodynamic, economic, compromised, and sustainable considerations can be suggested. Efficient power was introduced by Stucki ${ }^{149}$ while studying the mitochondrial energetic processes within the context of the linear non-equilibrium thermodynamics. Later the idea was extended by Yan and Chen ${ }^{65-66}$ to the regime of FTT and given the so-called name efficient power by Yilmaz. ${ }^{12-13} \mathrm{His}$ intention was to get a trade-off between the delivered power and the efficiency for a heat engine. He called this new function as "Efficient Power" introduce defined as the product of the power output by the efficiency: $P_{\eta}=P \eta$. It is shown that the efficient power criterion is also well suited to study the optimization of biological systems, ${ }^{139,149-151}$ steady and non-steady electric energy converters, ${ }^{152}$ thermionic generator, ${ }^{153}$ and low dissipation heat engines. ${ }^{154}$ For some naturally designed biological systems, Maximum Efficient Power (MEP) conditions may lead to more efficient engines 
than those at maximum Omega function $(M \Omega)$ or ecological function. ${ }^{151}$ It has been observed that the universal features of efficiency are not exclusive to the conditions of MP and $M \Omega$ but also extend to the engines operating in MEP regime also as shown below ${ }^{65}$

$\eta_{M E P}=\frac{2}{3} \eta_{c}+\frac{2}{27} \eta_{c}^{2}+O\left(\eta_{c}^{3}\right)$

Where in, the universality of linear term and quadratic term is related to tight-coupling condition and to left-right symmetry respectively as discussed for MP and ME criterion. These two terms in the above equation were also derived for the MEP of a nonlinear irreversible heat engine ${ }^{152}$ working in strong coupling limit under the symmetric condition by using master equation model. The low dissipation model with symmetric dissipation also displays similar behaviour (Eq. (63)) up to quadratic term while for asymmetric dissipation, the lower and upper bounds for the efficiency in MEP regime are derived. ${ }^{153}$

\section{Multi-parameter Combined Performance Criteria}

Yan ${ }^{65-66}$ introduce a multi-parameter combined objective function of the efficiency and power output as

$F=\eta^{\lambda} P$

where $\lambda$ is a weight factor whose value may be chosen from zero to infinity. The chosen parameter $\lambda$ here has explicit physical meaning, because every value of $\lambda$ corresponds to one particular function. This general multi-parameter combined objective function can be adopted as the optimization criterion instead of the single optimization criterion to derive the universal expression of the efficiency for a simple model of heat engines

In Ref.[151], by using stochastic thermodynamic analysis with a master equation description of a driven open system ${ }^{155}$, the universal expression of the optimization efficiency with a multi-parameter combined objective function is derived. The results obtained show that the optimization efficiency displays universality up to quadratic order term of $\eta_{c}$ for the strong coupling systems in presence of left-right symmetry. It has been shown that the optimal results derived from the multi-parameter combined objective function can be directly used to describe the performance of nonlinear irreversible heat engines operating at the maximum power $(\lambda=0)$, the maximum efficiency-power state $(\lambda=1)$, the maximum ecological or unified trade-off function $(\lambda=2)$, and the maximum efficiency $(\lambda=\infty)$. This study fully embodies the advantages of using the multi-parameter combined objective function to discuss the optimization problems of thermodynamic systems. ${ }^{156}$

\section{Discussion}

In this review paper, we had briefly discussed the optimization of heat engines under different objective functions since thermodynamic optimization has a crucial role in identifying the mechanism that provides optimal efficiency for finite-time processes. We discussed the key procedures to derive the optimization efficiencies at maximum power, maximum ecological (or Omega) function and maximum efficient power. First, we investigated the Efficiency at Maximum Power (EMP) for different models of heat engines. The universal behavior of EMP can be observed in the study of these models up to second order when expanded near-equilibrium. However, this universality seems to be broken down for the quantum dot engine. The origin of this universality breaks down lies in terms of irreversible thermodynamics and a singular behaviour of the mechanical current. In fact, the absence of linear response regime of thermodynamic fluxes may yield various values of the linear coefficient in the standpoint of irreversible thermodynamics. It has been noted that heat engines operating at MP are not the most efficient ones and, hence, are not much economical. Actual thermal plants and heat engines should not operate at MP, but in a regime with slightly smaller power and appreciable larger efficiency. ${ }^{157-158}$ The optimization of Omega criterion (ecological criterion) and efficient power criterion falls in such a regime. They pay equal attention to both power output and efficiency. Firstly, we investigated the efficiency at maximum $\Omega$ criterion (or ecological criterion) for the heat engine models considered for maximum power optimization. It was verified that the efficiency at maximum $\Omega$ criterion also exhibits universality up to quadratic order in the deviation from equilibrium for the strong coupling systems in the presence of a symmetry condition. Finally, optimization of another objective function named efficient power has been investigated and it also leads to universal behaviour of efficiency in different models. This condition is in agreement with that of the universal efficiency at maximum power. Then, a multi-parameter combined objective function is discussed which includes different objective functions is appearing in literature. Recently, it has been pointed out that the problem of local stability of operation regimes can be related with the regime's optimization itself. ${ }^{159-161}$

\section{Conclusion}

Concluding, we can say that, to achieve an engine's optimized performance, a suitable performance criterion has to be introduced and optimized. The use of various performance criteria leads to different performances in optimization and is suitable for different specific considerations. Although, there are many approaches to describe good performance regimes of heat engines but still there is no systematic method on which these criteria are based. We have the freedom to select any performance criterion based on thermodynamic, economic, compromised, and sustainable considerations. 


\section{Acknowledgment}

The author is thankful to Mr. Pranshu for his valuable suggestions and discussions in preparing this review paper.

\section{References}

1. Carnot, Sadi. "Réflexions sur la puissance motrice du feu et sur les machines propres à développer cette puissance." In Annales scientifiques École Normale Supérieure, vol. 1, pp. 393-457. 1872.

2. Kondepudi, Dilip, and Ilya Prigogine. Modern thermodynamics: from heat engines to dissipative structures. John Wiley \& Sons, 2014.

3. Curzon, Frank L., and B. Ahlborn. "Efficiency of a Carnot engine at maximum power output." American Journal of Physics 43, no. 1 (1975): 22-24.

4. Angulo-Brown, Fernando. "An ecological optimization criterion for finite-time heat engines." Journal of Applied Physics 69, no. 11 (1991): 7465-7469.

5. Sahin, Bahri, Ali Kodal, and Hasbi Yavuz. "Efficiency of a Joule-Brayton engine at maximum power density." Journal of Physics D: Applied Physics 28, no. 7 (1995): 1309.

6. Bejan, Adrian. "Entropy generation minimization: The new thermodynamics of finite-size devices and finite-time processes." Journal of Applied Physics 79, no. 3 (1996): 1191-1218.

7. Hernández, A. Calvo, A. Medina, J. M. M. Roco, J. A. White, and S. Velasco. "Unified optimization criterion for energy converters." Physical Review E 63, no. 3 (2001): 037102.

8. Durmayaz, Ahmet, Oguz Salim Sogut, Bahri Sahin, and Hasbi Yavuz. "Optimization of thermal systems based on finite-time thermodynamics and thermoeconomics." Progress in Energy and Combustion Science 30, no. 2 (2004): 175-217.

9. Izumida, Yuki, and Koji Okuda. "Work output and efficiency at maximum power of linear irreversible heat engines operating with a finite-sized heat source." Physical review letters 112, no. 18 (2014): 180603.

10. Allahverdyan, Armen E., Karen V. Hovhannisyan, Alexey V. Melkikh, and Sasun G. Gevorkian. "Carnot cycle at finite power: attainability of maximal efficiency." Physical review letters 111, no. 5 (2013): 050601.

11. Chen, Lingen, Chih Wu, and Fengrui Sun. "Finite time thermodynamic optimization or entropy generation minimization of energy systems." Journal of NonEquilibrium Thermodynamics 24, no. 4 (1999): 327359.

12. Yilmaz, T. "A new performance criterion for heat engines: efficient power." Journal of the Energy Institute 79, no. 1 (2006): 38-41.
13. Yilmaz, Tamer, and Yalçın Durmuşoğlu. "Efficient power analysis for an irreversible Carnot heat engine." International journal of energy research 32, no. 7 (2008): 623-628.

14. Apertet, Y., H. Ouerdane, C. Goupil, and Ph Lecoeur. “Revisiting Feynman's ratchet with thermoelectric transport theory." Physical Review E 90, no. 1 (2014): 012113.

15. Hoffmann, Karl Heinz, Josef Maximilian Burzler, and Sven Schubert. "Endoreversible thermodynamics." (1997).

16. Hoffmann, Karl Heinz. "An introduction to endoreversible thermodynamics." (2005).

17. Callen, Herbert B. "Thermodynamics and an Introduction to Thermostatistics." (1998): 164-167.

18. Gutkowicz-Krusin, Dina, Itamar Procaccia, and John Ross. "On the efficiency of rate processes. Power and efficiency of heat engines." The Journal of Chemical Physics 69, no. 9 (1978): 3898-3906.

19. Orlov, V. N. "Optimum irreversible Carnot cycle containing three isotherms." SPhD 20 (1985): 506.

20. De Vos, Alexis. "Efficiency of some heat engines at maximum-power conditions." American Journal of Physics 53, no. 6 (1985): 570-573.

21. De Vos, Alexis. "Reflections on the power delivered by endoreversible engines." Journal of Physics D: Applied Physics 20, no. 2 (1987): 232.

22. Chen, Lixuan, and Zijun Yan. "The effect of heattransfer law on performance of a two-heat-source endoreversible cycle." The Journal of Chemical Physics 90, no. 7 (1989): 3740-3743.

23. Yan, Zijun, and Jincan Chen. "A class of irreversible Carnot refrigeration cycles with a general heat transfer law." Journal of Physics D: Applied Physics 23, no. 2 (1990): 136.

24. Gordon, J. M. "Observations on efficiency of heat engines operating at maximum power." American Journal of Physics 58, no. 4 (1990): 370-375.

25. Wu, Chih, and Robert L. Kiang. "Finite-time thermodynamic analysis of a Carnot engine with internal irreversibility." Energy 17, no. 12 (1992): 1173-1178.

26. Van den Broeck, Christian. "Thermodynamic efficiency at maximum power." Physical review letters 95, no. 19 (2005): 190602.

27. Gomez-Marin, Alejandro, and José M. Sancho. "Tight coupling in thermal Brownian motors." Physical Review E 74, no. 6 (2006): 062102.

28. De Cisneros, B. Jiménez, and A. Calvo Hernández. "Collective working regimes for coupled heat engines." Physical review letters 98, no. 13 (2007): 130602

29. Schmiedl, Tim, and Udo Seifert. "Efficiency at 
maximum power: An analytically solvable model for stochastic heat engines." EPL (Europhysics Letters) 81, no. 2 (2007): 20003.

30. Tu, Z. C. "Efficiency at maximum power of Feynman's ratchet as a heat engine." Journal of Physics A: Mathematical and Theoretical 41, no. 31 (2008): 312003.

31. Esposito, Massimiliano, Katja Lindenberg, and Christian Van den Broeck. "Thermoelectric efficiency at maximum power in a quantum dot." EPL (Europhysics Letters) 85, no. 6 (2009): 60010.

32. Esposito, Massimiliano, Katja Lindenberg, and Christian Van den Broeck. "Universality of efficiency at maximum power." Physical review letters 102, no. 13 (2009): 130602.

33. Esposito, Massimiliano, Ryoichi Kawai, Katja Lindenberg, and Christian Van den Broeck. "Quantum-dot Carnot engine at maximum power." Physical review E 81, no. 4 (2010): 041106.

34. Esposito, Massimiliano, Ryoichi Kawai, Katja Lindenberg, and Christian Van den Broeck. "Efficiency at maximum power of low-dissipation Carnot engines." Physical review letters 105, no. 15 (2010): 150603.

35. Wang, Xian-Zhi. "Irreversible cycle in linear irreversible thermodynamics." Journal of Physics A: Mathematical and Theoretical 43, no. 42 (2010): 425003.

36. Abe, Sumiyoshi. "Maximum-power quantummechanical Carnot engine." Physical Review E 83, no. 4 (2011): 041117.

37. Wang, Yang, and Z. C. Tu. "Efficiency at maximum power output of linear irreversible Carnot-like heat engines." Physical Review E 85, no. 1 (2012): 011127.

38. Izumida, Yuki, and Koji Okuda. "Efficiency at maximum power of minimally nonlinear irreversible heat engines." EPL (Europhysics Letters) 97, no. 1 (2012): 10004.

39. Izumida, Y., K. Okuda, A. Calvo Hernández, and J. M. M. Roco. "Coefficient of performance under optimized figure of merit in minimally nonlinear irreversible refrigerator." EPL (Europhysics Letters) 101, no. 1 (2013): 10005.

40. Uzdin, Raam, and Ronnie Kosloff. "Universal features in the efficiency at maximal work of hot quantum otto engines." EPL (Europhysics Letters) 108, no. 4 (2014): 40001.

41. Sheng, Shiqi, and Z. C. Tu. "Constitutive relation for nonlinear response and universality of efficiency at maximum power for tight-coupling heat engines." Physical Review E 91, no. 2 (2015): 022136.

42. Cleuren, Bart, Bob Rutten, and C. Van den Broeck. "Universality of efficiency at maximum power." The
European Physical Journal Special Topics 224, no. 5 (2015): 879-889.

43. Whitney, Robert S. "Most efficient quantum thermoelectric at finite power output." Physical review letters 112, no. 13 (2014): 130601.

44. Whitney, Robert S. "Finding the quantum thermoelectric with maximal efficiency and minimal entropy production at given power output." Physical Review B 91, no. 11 (2015): 115425.

45. Holubec, Viktor, and Artem Ryabov. "Efficiency at and near maximum power of low-dissipation heat engines." Physical Review E 92, no. 5 (2015): 052125.

46. Holubec, Viktor, and Artem Ryabov. "Erratum: Efficiency at and near maximum power of lowdissipation heat engines [Phys. Rev. E 92, 052125 (2015)]." Physical Review E 93, no. 5 (2016): 059904.

47. Long, Rui, and Wei Liu. "Efficiency and its bounds of minimally nonlinear irreversible heat engines at arbitrary power." Physical Review E 94, no. 5 (2016): 052114.

48. Ryabov, Artem, and Viktor Holubec. "Maximum efficiency of steady-state heat engines at arbitrary power." Physical Review E 93, no. 5 (2016): 050101.

49. Dechant, Andreas, Nikolai Kiesel, and Eric Lutz. "Underdamped stochastic heat engine at maximum efficiency." EPL (Europhysics Letters) 119, no. 5 (2017): 50003.

50. Izumida, Yuki, and Koji Okuda. "Molecular kinetic analysis of a finite-time Carnot cycle." EPL (Europhysics Letters) 83, no. 6 (2008): 60003.

51. Izumida, Yuki, and Koji Okuda. "Onsager coefficients of a finite-time Carnot cycle." Physical review E 80, no. 2 (2009): 021121.

52. Allen, Michael P., and Dominic J. Tildesley. Computer simulation of liquids. Oxford university press, 2017.

53. Sadus, R. J. Molecular Simulation of Fluids: Theory, Algorithms and Object-Orientation. Elsevier, 1999.

54. Alder, Berni J., and Thomas Everett Wainwright. "Studies in molecular dynamics. I. General method." The Journal of Chemical Physics 31, no. 2 (1959): 459-466.

55. Yan, Z. "Comment on "Ecological optimization criterion for finite-time heat engines", Journal of Applied Physics 73, no. 7 (1993): 3583.

56. Zhang, Yanchao, Chuankun Huang, Guoxing Lin, and Jincan Chen. "Universality of efficiency at unified trade-off optimization." Physical Review E 93, no. 3 (2016): 032152.

57. Angulo-Brown, Fernando, M. Santillán, and E. Calleja-Quevedo. "Thermodynamic optimality in some biochemical reactions." II Nuovo Cimento D 17, no. 1 (1995): 87-90.

58. Arias, L. A. "Hernandez and F. Angulo-Brown." J. 
Appl. Phys 81 (1997): 2973.

59. Arias-Hernandez, Luis Antonio, Fernando AnguloBrown, and R. T. Paez-Hernandez. "First-order irreversible thermodynamic approach to a simple energy converter." Physical Review E 77, no. 1 (2008): 011123.

60. Sanchez-Salas, Norma, L. López-Palacios, S. Velasco, and A. Calvo Hernández. "Optimization criteria, bounds, and efficiencies of heat engines." Physical Review E 82, no. 5 (2010): 051101.

61. De Tomás, C., J. M. M. Roco, A. Calvo Hernández, Yang Wang, and Z. C. Tu. "Low-dissipation heat devices: Unified trade-off optimization and bounds." Physical Review E 87, no. 1 (2013): 012105.

62. Long, Rui, and Wei Liu. "Unified trade-off optimization for general heat devices with nonisothermal processes." Physical Review E 91, no. 4 (2015): 042127.

63. Long, Rui, Zhichun Liu, and Wei Liu. "Performance optimization of minimally nonlinear irreversible heat engines and refrigerators under a trade-off figure of merit." Physical Review E 89, no. 6 (2014): 062119.

64. Singh, Varinder, and Ramandeep S. Johal. "Threelevel laser heat engine at optimal performance with ecological function." Physical Review E 100, no. 1 (2019): 012138.

65. Yan, $Z$. " $\eta$ and $P$ of a Carnot engine at maximum $\eta$ $\lambda$ P, J." Xiamen Univ 25 (1986): 279-286.

66. Yan, Zijun, and Jincan Chen. "A generalized Rutgers formula derived from the theory of endoreversible cycles." Physics Letters A 217, no. 2-3 (1996): 137140.

67. Maheshwari, Govind, S. Chaudhary, and S. K. Somani. "Performance analysis of a generalized radiative heat engine based on new maximum efficient power approach." International Journal of Low-Carbon Technologies 4, no. 1 (2009): 9-15.

68. Maheshwari, Govind, S. Chaudhary, and S. K. Somani. "Performance analysis of endoreversible combined Carnot cycles based on new maximum efficient power (MEP) approach." International Journal of Low-Carbon Technologies 5, no. 1 (2010): 1-6.

69. Singh, Varinder, and Ramandeep S. Johal. "Lowdissipation Carnot-like heat engines at maximum efficient power." Physical Review E 98, no. 6 (2018): 062132.

70. Zhan-Chun, Tu. "Recent advance on the efficiency at maximum power of heat engines." Chinese Physics B 21, no. 2 (2012): 020513.

71. Novikov, I. I. "The efficiency of atomic power stations (A review)." Atommaya Energiya, no. 11 (1957), 409.

72. Chambadal, Paul. "Las centrales nucleares." Manuales/Ingenieria (1957).
73. Andresen, Bjarne, Peter Salamon, and R. Stephen Berry. "Thermodynamics in finite time: extremals for imperfect heat engines." The Journal of Chemical Physics 66, no. 4 (1977): 1571-1577.

74. Rubin, Morton H. "Optimal configuration of a class of irreversible heat engines. I." Physical Review A 19, no. 3 (1979): 1272.

75. Salamon, Peter, and Abrahan Nitzan. "Finite time optimizations of a Newton's law Carnot cycle." The Journal of Chemical Physics 74, no. 6 (1981): 35463560.

76. Bejan, Adrian. "Models of power plants that generate minimum entropy while operating at maximum power." American Journal of Physics 64, no. 8 (1996): 1054-1059.

77. Orlov, Vladimir N., and R. Stephen Berry. "Analytical and numerical estimates of efficiency for an irreversible heat engine with distributed working fluid.” Physical Review A 45, no. 10 (1992): 7202.

78. Chen, L., Sun, F., Chen, W. "Optimal expansion of a heated working fluid in a cylinder with a movable piston in the case of $q \Delta\left(T^{\wedge}(-1)\right) s^{\prime \prime}$. Chinese J. Mech. Engng. 29, (1993):97

79. Angulo-Brown, Fernando, and R. Páez-Hernández. "Endoreversible thermal cycle with a nonlinear heat transfer law." Journal of applied physics 74, no. 4 (1993): 2216-2219.

80. Yan, Zijun, and Jincan Chen. "A class of irreversible Carnot refrigeration cycles with a general heat transfer law." Journal of Physics D: Applied Physics 23, no. 2 (1990): 136.

81. Chen, W. Z., F. R. Sun, and L. G. Chen. "The optimal relation of a carnot heat pump for the case $\mathrm{Q} \Delta \mathrm{T} \_\mathrm{n}$." In Proceedings of the International Energy Conference on Energy Conversion and Energy Source Engineering, pp. 18-20. 1990.

82. Chen, W. Z., F. R. Sun, S. M. Cheng, and L. G. Chen. "Study on optimal performance and working temperatures of endoreversible forward and reverse Carnot cycles." International journal of energy research 19, no. 9 (1995): 751-759.

83. Nulton, James D., Peter Salamon, and Raj K. Pathria. "Carnot-like processes in finite time. I. Theoretical limits." American Journal of Physics 61, no. 10 (1993): 911-916.

84. Sekimoto, Ken. "Kinetic characterization of heat bath and the energetics of thermal ratchet models." Journal of the physical society of Japan 66 , no. 5 (1997): 1234-1237.

85. Seifert, Udo. "Entropy production along a stochastic trajectory and an integral fluctuation theorem." Physical review letters 95, no. 4 (2005): 040602.

86. Schmiedl, Tim, and Udo Seifert. "Optimal finite-time 
processes in stochastic thermodynamics." Physical review letters 98, no. 10 (2007): 108301.

87. Park, Jong-Min, Hyun-Myung Chun, and Jae Dong Noh. "Efficiency at maximum power and efficiency fluctuations in a linear Brownian heat-engine model." Physical Review E 94, no. 1 (2016): 012127.

88. Feynman, Richard Phillips, Robert B. Leighton, and Matthew Sands. Lições de física: the Feynman lectures on physics. Bookman, 2008.

89. Jarzynski, C., and O. Mazonka. "Feynman's ratchet and pawl: An exactly solvable model." Physical Review E 59, no. 6 (1999): 6448.

90. Velasco, S., J. M. M. Roco, A. Medina, and A. Calvo Hernández. "Feynman's ratchet optimization: maximum power and maximum efficiency regimes." Journal of Physics D: Applied Physics 34, no. 6 (2001): 1000.

91. Zhang, Y., B. H. Lin, and J. C. Chen. "Performance characteristics of an irreversible thermally driven Brownian microscopic heat engine." The European Physical Journal B-Condensed Matter and Complex Systems 53, no. 4 (2006): 481-485.

92. Ai, B-Q., H-Z. Xie, D-H. Wen, X-M. Liu, and L-G. Liu. "Heat flow and efficiency in a microscopic engine." The European Physical Journal B-Condensed Matter and Complex Systems 48, no. 1 (2005): 101-106.

93. Parrondo, Juan MR, and Pep Español. "Criticism of Feynman's analysis of the ratchet as an engine." American Journal of Physics 64, no. 9 (1996): 11251130.

94. Derényi, Imre, and R. Dean Astumian. "Efficiency of Brownian heat engines." Physical Review E 59, no. 6 (1999): R6219.

95. Chen, Lingen, Zemin Ding, and Fengrui Sun. "Optimum performance analysis of Feynman's engine as cold and hot ratchets." Journal of NonEquilibrium Thermodynamics 36, no. 2 (2011): 155177.

96. Apertet, Y., H. Ouerdane, C. Goupil, and Ph Lecoeur. "Efficiency at maximum power of thermally coupled heat engines." Physical Review E 85, no. 4 (2012): 041144.

97. Smoluchowski, Marian. "Experimentell nachweisbare, der üblichen Thermodynamik widersprechende Molekularphänomene." Pisma Mariana Smoluchowskiego 2, no. 1 (1927): 226-251.

98. Singh, Varinder, and Ramandeep S. Johal. "FeynmanSmoluchowski engine at high temperatures and the role of constraints." Journal of Statistical Mechanics: Theory and Experiment 2018, no. 7 (2018): 073205.

99. Van den Broeck, Christian, and Katja Lindenberg. "Efficiency at maximum power for classical particle transport." Physical Review E 86, no. 4 (2012):
041144.

100. Abah, Obinna, Johannes Rossnagel, Georg Jacob, Sebastian Deffner, Ferdinand Schmidt-Kaler, Kilian Singer, and Eric Lutz. "Single-ion heat engine at maximum power." Physical review letters 109, no. 20 (2012): 203006.

101. Wang, Rui, Jianhui Wang, Jizhou He, and Yongli Ma. "Efficiency at maximum power of a heat engine working with a two-level atomic system." Physical Review E 87, no. 4 (2013): 042119.

102. Wu, Feilong, Jizhou He, Yongli Ma, and Jianhui Wang. "Efficiency at maximum power of a quantum Otto cycle within finite-time or irreversible thermodynamics." Physical Review E 90, no. 6 (2014): 062134.

103. De Vos, Alexis. "Efficiency of some heat engines at maximum-power conditions." American Journal of Physics 53, no. 6 (1985): 570-573.

104. Rubin, Morton H., and Bjarne Andresen. "Optimal staging of endoreversible heat engines." Journal of Applied Physics 53, no. 1 (1982): 1-7.

105. Van den Broeck, C. "Efficiency at maximum power in the low-dissipation limit." EPL (Europhysics Letters) 101, no. 1 (2013): 10006.

106. Aurell, Erik, Krzysztof Gawędzki, Carlos MejíaMonasterio, Roya Mohayaee, and Paolo Muratore-Ginanneschi. "Refined second law of thermodynamics for fast random processes." Journal of statistical physics 147, no. 3 (2012): 487-505.

107. Sekimoto, Ken, and Shin-ichi Sasa. "Complementarity relation for irreversible process derived from stochastic energetics." Journal of the Physical Society of Japan 66, no. 11 (1997): 3326-3328.

108. Sekimoto, Ken, and Shin-ichi Sasa. "Complementarity relation for irreversible process derived from stochastic energetics." Journal of the Physical Society of Japan 66, no. 11 (1997): 3326-3328.

109. Wang, Yang, and Z. C. Tu. "Efficiency at maximum power output of linear irreversible Carnot-like heat engines." Physical Review E 85, no. 1 (2012): 011127.

110. Guo, Juncheng, Junyi Wang, Yuan Wang, and Jincan Chen. "Universal efficiency bounds of weakdissipative thermodynamic cycles at the maximum power output." Physical Review E 87, no. 1 (2013): 012133.

111. Sheng, Shiqi, and Z. C. Tu. "Universality of energy conversion efficiency for optimal tight-coupling heat engines and refrigerators." Journal of Physics A: Mathematical and Theoretical 46, no. 40 (2013): 402001.

112. Sheng, Shiqi, and Z. C. Tu. "Weighted reciprocal of temperature, weighted thermal flux, and their applications in finite-time thermodynamics." Physical 
Review E 89, no. 1 (2014): 012129.

113. Benenti, Giuliano, Keiji Saito, and Giulio Casati. "Thermodynamic bounds on efficiency for systems with broken time-reversal symmetry." Physical review letters 106, no. 23 (2011): 230602.

114. Iyyappan, I., and Ramandeep S. Johal. "Efficiency of a two-stage heat engine at optimal power." EPL (Europhysics Letters) 128, no. 5 (2020): 50004.

115. Nakpathomkun, Natthapon, Hongqi Q. Xu, and Heiner Linke. "Thermoelectric efficiency at maximum power in low-dimensional systems." Physical Review B 82, no. 23 (2010): 235428.

116. Kennes, D. M., D. Schuricht, and V. Meden. "Efficiency and power of a thermoelectric quantum dot device." EPL (Europhysics Letters) 102, no. 5 (2013): 57003.

117. Sothmann, Björn, Rafael Sánchez, and Andrew N. Jordan. "Thermoelectric energy harvesting with quantum dots." Nanotechnology 26, no. 3 (2014): 032001.

118. Lee, Sang Hoon, Jaegon Um, and Hyunggyu Park. "Nonuniversality of heat-engine efficiency at maximum power." Physical Review E 98, no. 5 (2018): 052137.

119. Josefsson, Martin, Artis Svilans, Heiner Linke, and Martin Leijnse. "Optimal power and efficiency of single quantum dot heat engines: Theory and experiment." Physical Review B 99, no. 23 (2019): 235432

120. Du, Jianying, Wei Shen, Xin Zhang, Shanhe Su, and Jincan Chen. "Quantum-dot heat engines with irreversible heat transfer." Physical Review Research 2, no. 1 (2020): 013259.

121. Bonet, Edgar, Mandar M. Deshmukh, and D. C. Ralph. "Solving rate equations for electron tunneling via discrete quantum states." Physical Review B 65, no. 4 (2002): 045317.

122. Bagrets, D. A., and Yu V. Nazarov. "Full counting statistics of charge transfer in Coulomb blockade systems." Physical Review B 67, no. 8 (2003): 085316.

123. Harbola, Upendra, Massimiliano Esposito, and Shaul Mukamel. "Quantum master equation for electron transport through quantum dots and single molecules." Physical Review B 74, no. 23 (2006): 235309.

124. Sheng, Shiqi, and Z. C. Tu. "Weighted reciprocal of temperature, weighted thermal flux, and their applications in finite-time thermodynamics." Physical Review E 89, no. 1 (2014): 012129.

125. Sheng, Shiqi, and Z. C. Tu. "Constitutive relation for nonlinear response and universality of efficiency at maximum power for tight-coupling heat engines." Physical Review E 91, no. 2 (2015): 022136.

126. De Groot, S. R., and P. Mazur. "Dover." New York
(1984): 280-281.

127. Gaveau, B., M. Moreau, and L. S. Schulman. "Stochastic thermodynamics and sustainable efficiency in work production." Physical review letters 105, no. 6 (2010): 060601.

128. Holubec, Viktor, and Artem Ryabov. "Maximum efficiency of low-dissipation heat engines at arbitrary power." Journal of Statistical Mechanics: Theory and Experiment 2016, no. 7 (2016): 073204.

129. Salamon, Peter, Abraham Nitzan, Bjarne Andresen, and R. Stephen Berry. "Minimum entropy production and the optimization of heat engines." Physical Review A 21, no. 6 (1980): 2115.

130. Cheng, Ching-Yang. "The ecological optimization of an irreversible Carnot heat engine." Journal of physics D: Applied physics 30, no. 11 (1997): 1602.

131. Yan, Zijun, and Guoxing Lin. "Ecological optimization criterion for an irreversible three-heat-source refrigerator." Applied energy 66, no. 3 (2000): 213224.

132. Salas, N. Sánchez, and A. Calvo Hernández. "Unified working regime of nonlinear systems rectifying thermal fluctuations." EPL (Europhysics Letters) 61, no. 3 (2003): 287.

133. Chen, Lingen, Jianping Zhou, Fengrui Sun, and Chih Wu. "Ecological optimization for generalized irreversible Carnot engines." Applied Energy 77, no. 3 (2004): 327-338.

134. De Cisneros, B. Jiménez, and A. Calvo Hernández. "Collective working regimes for coupled heat engines." Physical review letters 98, no. 13 (2007): 130602.

135. 135. De Cisneros, B. Jiménez, and A. Calvo Hernández. "Coupled heat devices in linear irreversible thermodynamics." Physical Review E 77, no. 4 (2008): 041127.

136. Long, Rui, and Wei Liu. "Ecological optimization for general heat engines." Physica A: Statistical Mechanics and its Applications 434 (2015): 232-239.

137. Özel, Gülcan, Emin Açıkkalp, Ahmet Fevzi Savaş, and Hasan Yamık. "Comparative analysis of thermoeconomic evaluation criteria for an actual heat engine." Journal of Non-Equilibrium Thermodynamics 41, no. 3 (2016): 225-235.

138. Sanchez-Salas, Norma, and A. Calvo Hernández. "Harmonic quantum heat devices: Optimumperformance regimes." Physical Review E 70, no. 4 (2004): 046134.

139. Arias-Hernandez, Luis Antonio, Fernando AnguloBrown, and R. T. Paez-Hernandez. "First-order irreversible thermodynamic approach to a simple energy converter." Physical Review E 77, no. 1 (2008): 011123. 
140. Long, Rui, Zhichun Liu, and Wei Liu. "Performance optimization of minimally nonlinear irreversible heat engines and refrigerators under a trade-off figure of merit." Physical Review E 89, no. 6 (2014): 062119.

141. Landsberg, Peter T., and Harvey S. Leff. "Thermodynamic cycles with nearly universal maximum-work efficiencies." Journal of Physics A: Mathematical and General 22, no. 18 (1989): 4019.

142. Kosloff, Ronnie. "A quantum mechanical open system as a model of a heat engine." The Journal of chemical physics 80, no. 4 (1984): 1625-1631.

143. Bekele, Mulugeta, Mehari Bayou, Yergou Tatek, and Mesfin Tsige. "Optimizing the performance of a heat engine: A simulation study." APS 2011 (2011): V31-004.

144. Yuge, Tatsuro, Nobuyasu Ito, and Akira Shimizu. "Nonequilibrium molecular dynamics simulation of electric conduction." Journal of the Physical Society of Japan 74, no. 7 (2005): 1895-1898.

145. Hondou, Tsuyoshi. "Equation of state in a small system: Violation of an assumption of Maxwell's demon." EPL (Europhysics Letters) 80, no. 5 (2007): 50001.

146. Rojas-Gamboa, David A., Juan I. Rodríguez, Julian Gonzalez-Ayala, and F. Angulo-Brown. "Ecological efficiency of finite-time thermodynamics: A molecular dynamics study." Physical Review E 98, no. 2 (2018): 022130.

147. Angulo-Brown, Fernando, G. Ares de Parga, and Luis Antonio Arias-Hernandez. "A variational approach to ecological-type optimization criteria for finitetime thermal engine models." Journal of Physics D: Applied Physics 35, no. 10 (2002): 1089.

148. Tadele, Kumneger, Yergou B. Tatek, and Mulugeta Bekele. "Energetics of a heat engine: a molecular dynamics simulation study." European Physical Journal. Applied Physics 76, no. 2 (2016).

149. Stucki, Jörg W. "The optimal efficiency and the economic degrees of coupling of oxidative phosphorylation." European Journal of Biochemistry 109, no. 1 (1980): 269-283.

150. Chimal, J. C., N. Sánchez, and P. R. Ramírez. "Thermodynamic Optimality criteria for biological systems in linear irreversible thermodynamics." In Journal of Physics: Conference Series, vol. 792, no. 1, p. 012082. IOP Publishing, 2017.

151. Zhang, Yanchao, Juncheng Guo, Guoxing Lin, and Jincan Chen. "Universal optimization efficiency for nonlinear irreversible heat engines." Journal of Non-Equilibrium Thermodynamics 42, no. 3 (2017): 253-263.

152. Valencia-Ortega, Gabriel, and Luis-Antonio AriasHernandez. "Thermodynamic optimization of an electric circuit as a non-steady energy converter." Journal of Non-Equilibrium Thermodynamics 42, no. 2 (2017): 187-199.

153. Chen, Lingen, Zemin Ding, Junle Zhou, Wenhua Wang, and Fengrui Sun. "Thermodynamic performance optimization for an irreversible vacuum thermionic generator." The European Physical Journal Plus 132, no. 7 (2017): 1-12.

154. Singh, Varinder, and Ramandeep S. Johal. "Lowdissipation Carnot-like heat engines at maximum efficient power." Physical Review E 98, no. 6 (2018): 062132.

155. Oliveira, Mário J. de. "Classical and quantum stochastic thermodynamics." Revista Brasileira de Ensino de Física 42 (2020).

156. Ding, Zemin, Lingen Chen, and Xiaowei Liu. "Thermodynamic optimization for irreversible thermal Brownian motors, energy selective electron engines and thermionic devices." International Journal of Ambient Energy (2018): 1-5.

157. De Vos, Alexis. Endoreversible Thermodynamics of Solar Energy Conversion. Oxford University Press, 1992.

158. Chen, Jincan, Zijun Yan, Guoxing Lin, and Bjarne Andresen. "On the Curzon-Ahlborn efficiency and its connection with the efficiencies of real heat engines." Energy Conversion and Management 42, no. 2 (2001): 173-181.

159. Reyes-Ramírez, I., J. Gonzalez-Ayala, A. Calvo Hernández, and M. Santillán. "Local-stability analysis of a low-dissipation heat engine working at maximum power output." Physical Review E 96, no. 4 (2017): 042128.

160. Gonzalez-Ayala, Julián, Moisés Santillán, Israel Reyes-Ramírez, and Antonio Calvo-Hernández. "Link between optimization and local stability of a low-dissipation heat engine: Dynamic and energetic behaviors." Physical Review E 98, no. 3 (2018): 032142.

161. Gonzalez-Ayala, Julian, Moises Santillán, Maria Jesus Santos, Antonio Calvo Hernández, and Jose Miguel Mateos Roco. "Optimization and stability of heat engines: The role of entropy evolution." Entropy 20, no. 11 (2018): 865. 